Valentin Blomer

\title{
On the 4-norm of an automorphic form
}

Received October 26, 2011 and in revised form January 30, 2012

Abstract. We prove the optimal upper bound $\sum_{f}\|f\|_{4}^{4} \ll q^{\varepsilon}$ where $f$ runs over an orthonormal basis of Maaß cusp forms of prime level $q$ and bounded spectral parameter.

Keywords. $L^{p}$-norm, triple product $L$-functions, character sums

\section{Introduction}

Bounding $L^{p}$-norms of functions on a Riemannian surface (for $2<p \leq \infty$ ) can be regarded as a weak type of equidistribution statement. The situation is particularly interesting for manifolds with additional symmetries, such as a commutative algebra of Hecke operators commuting with the Laplacian, among other things because one can consider joint eigenfunctions which may rule out high multiplicity of eigenspaces. Often the underlying manifold is kept fixed, and one searches for bounds in terms of the Laplacian eigenvalue $\lambda$ as $\lambda \rightarrow \infty$. Here the first breakthrough for an arithmetic hyperbolic surface in the case $p=\infty$ has been obtained by Iwaniec and Sarnak [IS].

In this article we change the point of view and keep the spectral data fixed, but study instead the dependence on the manifold. We are interested in the 4-norm of a Maaß form on a hyperbolic surface $X_{0}(q):=\Gamma_{0}(q) \backslash \mathbb{H}$ where $q$ is a large prime. Equipped with the inner product

$$
\langle f, g\rangle=\int_{X_{0}(q)} f(z) \bar{g}(z) \frac{d x d y}{y^{2}},
$$

the space $X_{0}(q)$ has volume

$$
V(q):=\operatorname{vol}\left(X_{0}(q)\right)=\frac{\pi}{3}(q+1) .
$$

The 4-norm is a particularly interesting object because it is connected to triple product $L$-functions; by Watson's formula one has an equality roughly of the type

$$
\|f\|_{4}^{4} \approx \frac{1}{q^{2}} \sum_{t_{g} \ll 1} L(1 / 2, f \times \bar{f} \times g)
$$

V. Blomer: Mathematisches Institut, Bunsenstr. 3-5, 37073 Göttingen, Germany; e-mail: blomer@uni-math.gwdg.de

Mathematics Subject Classification (2010): 11F12, 11F67 
where the sum runs over an orthonormal basis of Hecke eigenforms $g$ of level $q$ with bounded spectral parameter $t_{g}$ (see (2.1) below). By Weyl's law, the sum on the right hand side of (1.3) has $O(q)$ terms, so the Lindelöf hypothesis for the $L$-functions on the right hand side of (1.3) would imply $\|f\|_{4} \ll q^{-1 / 4+\varepsilon}$, and this is best possible by (1.2).

The same type of period formula is also the starting point for bounding the 4-norm in the eigenvalue aspect, and in this case Sarnak and Watson have announced a complete solution (possibly under the Ramanujan conjecture). Often in the theory of automorphic forms the archimedean and non-archimedean parameters behave, at least on a large scale, similarly. In the context of bounding 4-norms, however, the spectral, weight and level aspects behave very differently: in spectral $t$ aspect, Watson's formula produces a sum of length $t^{2}$ of central $L$-values of conductor $t^{8}$, while in the weight $k$ aspect, one obtains a sum of length $k$ and conductor $k^{6}$, which is much harder to treat. The level aspect, which we focus on here, is also more difficult than the spectral aspect: the conductor of the $L$ functions in (1.3) is $q^{5}$, so again there is little hope to establish a Lindelöf-type bound unconditionally with present technology. The aim of this article is to confirm this bound on average over Maaß forms $f$ of level $q$ :

Theorem 1. Fix any real number $T>1$ and any $\varepsilon>0$. Then

$$
\sum_{t_{f} \leq T}\|f\|_{4}^{4} \ll_{T, \varepsilon} q^{\varepsilon}
$$

where the sum runs over an orthonormal basis of Maaß cusp forms of prime level $q$ and spectral parameter $t_{f} \leq T$.

Up to the power $q^{\varepsilon}$, Theorem 1 is best possible. For an individual form $f$, we have the trivial bound $\|f\|_{4} \leq\|f\|_{2}^{1 / 2}\|f\|_{\infty}^{1 / 2}$. Non-trivial estimates for $\|f\|_{\infty}$ have been obtained first in $[\mathrm{BH}]$, and the strongest result $[\mathrm{HT}]$ implies

$$
\|f\|_{4} \leq q^{-1 / 12+\varepsilon}
$$

for an $L^{2}$-normalized Maaß form. It seems hard to improve this on the basis of (1.3). Theorem 1 implies immediately the best possible bound $\|f\|_{4} \ll q^{-1 / 4+\varepsilon}$ for almost all $f$ :

Corollary 1. For any $\delta>0$ the bound $\|f\|_{4} \ll q^{-1 / 4+\delta}$ holds for all but $O\left(q^{1-4 \delta+\varepsilon}\right)$ of all Maaß forms $f$ occurring in the sum in (1.4).

Remark. Since we are implicitly comparing different spaces $X_{0}(q)$, it may be more elegant to express everything in terms of the probability measure

$$
\langle f, g\rangle_{\text {prob }}:=\frac{3}{\pi(q+1)} \int_{X_{0}(q)} f(z) \bar{g}(z) \frac{d x d y}{y^{2}} .
$$

With this normalization our results state that $\|f\|_{4} \ll q^{\varepsilon}$ on average over $f$ (or for almost all $f$ ) satisfying $\langle f, f\rangle_{\text {prob }}=1$. However, most of the quoted literature uses the inner product (1.1), and it is therefore more convenient to keep the traditional normalization. 
The bound of Theorem 1 holds also for holomorphic cusp forms $f \in S_{k}(q)$ of any (fixed) weight $k \geq 2$ and large prime level $q$. If $k$ is sufficiently large, one can use the Petersson formula instead of the Kuznetsov formula. For small $k$, one can isometrically embed the holomorphic spectrum of weight $k$ into the Maaß spectrum of weight $k$ and use an appropriate weight $k$ Kuznetsov formula (see [DFI]).

We remark by the way that the proof of Theorem 1 depends on moderately strong bounds towards the Ramanujan conjecture. Any bound $\left|\Re \mu_{\pi}(q, i)\right| \leq 1 / 2-\delta$ for the Langlands parameters associated to a cuspidal representation on $\pi$ on $\mathrm{GL}_{2}$ at the (unramified) place $v=q$, as well as the archimedean bound $\left|\Re \mu_{\pi}(\infty, i)\right| \leq 1 / 2-\delta$ for $\pi$ on $\mathrm{GL}_{2}$ and $\mathrm{GL}_{6}$ suffices. Alternatively, if one prefers to stay entirely in $\mathrm{GL}_{2}$, then $\left|\Re \mu_{\pi}(\infty, i)\right| \leq 1 / 6-\delta$ for $\pi$ on $\mathrm{GL}_{2}$ suffices. In addition, we use several deep facts such as the automorphy of $\mathrm{GL}_{2} \times \mathrm{GL}_{3} L$-functions [KSh], non-negativity of central values [La], and of course Watson's formula [Wa].

It follows from the period formula (1.3) that the sum on the left hand side of Theorem 1 is roughly given by

$$
q^{-2} \sum_{t_{f}, t_{g} \ll 1} L(1 / 2, f \times \bar{f} \times g) .
$$

The (seemingly) similar average $\sum_{f, g} L(1 / 2, f \times g \times h)$ for $f, g, h \in S_{2}(q)$ holomorphic forms of weight 2 and level $q$ has been studied in [FW], also on the basis of triple product identities, but using entirely different techniques.

There is another period formula in which the triple product $L$-functions in (1.5) occur, namely as restrictions of certain Yoshida lifts. Given two holomorphic cuspidal Hecke forms ${ }^{1} h_{1}, h_{2} \in S_{2}(q)$, one can define the (second) Yoshida lift $Y^{(2)}\left(h_{1}, h_{2}\right)$ which is a Siegel modular form of degree 2 and weight 2 . When restricted to the diagonal $\left({ }^{z_{1}} z_{2}\right)$, it is a modular form of weight 2 both in $z_{1}$ and $z_{2}$, and hence

$$
\begin{aligned}
& Y^{(2)}\left(h_{1}, h_{2}\right)\left(\begin{array}{ll}
z_{1} & z_{2}
\end{array}\right)=\sum_{f_{1}, f_{2} \in S_{2}(q)} c\left(f_{1}, f_{2}\right) \overline{f_{1}\left(z_{1}\right) f_{2}\left(z_{2}\right)}, \\
& c\left(f_{1}, f_{2}\right)=\int_{X_{0}(q)} \int_{X_{0}(q)} Y^{(2)}\left(h_{1}, h_{2}\right)\left({ }^{z_{1}}{ }_{z_{2}}\right) f\left(z_{1}\right) f\left(z_{2}\right) \frac{d x_{1} d y_{1}}{y_{1}^{2}} \frac{d x_{2} d y_{2}}{y_{2}^{2}} .
\end{aligned}
$$

A special case of a beautiful formula of Böcherer, Furusawa and Schulze-Pillot [BFSP, Corollary 2.7b] shows that for $h_{1}=h_{2}=h$ and $f_{1}=f_{2}=f$ the coefficient $c(f, f)$ is proportional to the central $L$-value $L(1 / 2, f \times f \times h)$. The quantity estimated in Theorem 1 can then be interpreted as the trace of the matrix $\left(c\left(f_{1}, f_{2}\right)\right)$, averaged over cusp forms $h$.

The paper is organized as follows: Sections $2-4$ and 7 contain auxiliary material on automorphic forms, $L$-functions, character sums and integrals of Bessel functions. In particular we provide computations with oldforms, newforms and Eisenstein series, a special type of approximate functional equation for the $L$-functions in question, and bounds for certain complete exponential sums and oscillating integrals that occur later in the analysis. Considerable difficulty comes from the fact that we are summing over a family

\footnotetext{
1 More general assumptions on levels and weights are possible, but the holomorphy assumption cannot easily be dropped.
} 
of $L$-functions with oscillating root number. Section 5 contains the main transformation from the average of 4-norms into smooth sums over products of Kloosterman sums that are estimated in Section 8.

\section{Fourier expansions}

The spectrum of $L^{2}\left(X_{0}(q)\right)$ consists of the constant function, Maaß forms, and Eisenstein series $E_{\infty}(\cdot, 1 / 2+i t), E_{0}(\cdot, 1 / 2+i t)$ for $t \in \mathbb{R}$, corresponding to the two $\left(\Gamma_{0}(q)\right.$ equivalence classes of) cusps $\mathfrak{a}=\infty, 0$. For any Maaß form $g$ we denote by

$$
t_{g}=\sqrt{\lambda_{g}-1 / 4} \in \mathcal{T}:=\mathbb{R} \cup(-1 / 2,1 / 2) i
$$

its spectral parameter.

Let $\mathcal{B}_{q}$ be an orthonormal basis of cuspidal Hecke-Maaß newforms for $\Gamma_{0}(q)$. Let $\mathcal{B}_{1}$ be a basis of Hecke-Maaß cusp forms for $\mathrm{SL}_{2}(\mathbb{Z})$ that is orthonormal with respect to the inner product (1.1). In particular, for $g \in \mathcal{B}_{1}$ one has trivially

$$
\|g\|_{\infty} \ll_{t_{g}} q^{-1 / 2}
$$

by (1.2). The implied constant depends polynomially on $t_{g}$, for instance $\left(1+\left|t_{g}\right|\right)^{1 / 4}$ is admissible.

For any such Hecke-Maaß cusp form $g$ in $\mathcal{B}_{q}$ or $\mathcal{B}_{1}$ we write $\lambda_{g}(n)$ for the $n$-th Hecke eigenvalue, and we put $\delta_{g}=0$ if $g$ is even and $\delta_{g}=1$ if $g$ is odd.

Newforms $g \in \mathcal{B}_{q}$ have two properties that we need later: they are eigenfunctions of the Fricke involution $z \mapsto-1 /(q z)$, and one has

$$
\lambda_{g}(q)= \pm q^{-1 / 2} .
$$

By Weyl's law we have

$$
\#\left\{g \in \mathcal{B}_{1} \mid t_{g} \leq T\right\} \ll T^{2}, \quad \#\left\{g \in \mathcal{B}_{q} \mid t_{g} \leq T\right\} \ll q T^{2} .
$$

For $g \in \mathcal{B}_{1}$ define

$$
g_{q}(z):=\left(1-\frac{q \lambda_{g}^{2}(q)}{(q+1)^{2}}\right)^{-1 / 2}\left(g(q z)-\frac{\lambda_{g}(q) q^{1 / 2}}{q+1} g(z)\right) .
$$

By [ILS, Proposition 2.6], $g$ and $g_{q}$ have the same norm and are orthogonal to each other. We conclude that

$$
\mathcal{B}:=\mathcal{B}_{q} \cup \mathcal{B}_{1} \cup \mathcal{B}_{1}^{*}, \quad \mathcal{B}_{1}^{*}:=\left\{g_{q} \mid g \in \mathcal{B}_{1}\right\},
$$

is an orthonormal basis (with respect to (1.1)) of the non-trivial cuspidal spectrum of $L^{2}\left(X_{0}(q)\right)$.

Let

$$
g(z)=\rho_{g}(1) \sum_{n \neq 0} \lambda_{g}(n) \sqrt{y} K_{i t_{g}}(2 \pi|n| y) e(n x)
$$


be the Fourier expansion of some $g$ in $\mathcal{B}_{1}$ or $\mathcal{B}_{q}$ where $\lambda_{g}(-n)= \pm \lambda_{g}(n)$ depending on whether $g$ is even or odd. We have the Rankin-Selberg bound

$$
\sum_{n \leq x}\left|\lambda_{g}(n)\right|^{2} \ll x\left(q\left(1+\left|t_{g}\right|\right) x\right)^{\varepsilon}
$$

and the individual bound

$$
\lambda_{g}(n) \ll n^{1 / 2-\delta}
$$

for some $\delta>0$. Since res rel $_{s=1} E_{\infty}(z, s)=V(q)^{-1}$, we can compute

$$
\begin{aligned}
1 & =\|g\|_{2}^{2}=V(q) \underset{s=1}{\operatorname{res}} \int_{X_{0}(q)}|g(z)|^{2} E_{\infty}(z, s) \frac{d x d y}{y^{2}} \\
& =\left|\rho_{g}(1)\right|^{2} V(q) \underset{s=1}{\operatorname{res}} \sum_{n \neq 0} \frac{\left|\lambda_{g}(n)\right|^{2}}{|n|^{s}} \int_{0}^{\infty} K_{i t_{g}}(2 \pi y)^{2} y^{s} \frac{d y}{y} \\
& =\left|\rho_{g}(1)\right|^{2} V(q) 2 L\left(1, \operatorname{Ad}^{2} g\right) \frac{\pi}{8 \cosh \left(\pi t_{g}\right)} \begin{cases}\zeta^{(q)}(2)^{-1}, & g \in \mathcal{B}_{q}, \\
\zeta(2)^{-1}, & g \in \mathcal{B}_{1} .\end{cases}
\end{aligned}
$$

We conclude

$$
\left|\rho_{g}(1)\right|=\left(\frac{2 \cosh \left(\pi t_{g}\right)}{L\left(1, \operatorname{Ad}^{2} g\right)}\right)^{1 / 2} \times \begin{cases}(q+1)^{-1 / 2}, & g \in \mathcal{B}_{1}, \\ \left(q^{\prime}\right)^{-1 / 2}, & g \in \mathcal{B}_{q},\end{cases}
$$

where

$$
q^{\prime}:=\frac{q^{2}}{q-1} \asymp q .
$$

Let $g \in \mathcal{B}_{1}$ and let us define

$$
\lambda_{g}^{*}(n):=\left(1-\frac{q \lambda_{g}^{2}(q)}{(q+1)^{2}}\right)^{-1 / 2}\left(q^{1 / 2} \lambda_{g}\left(\frac{n}{q}\right)-\frac{\lambda_{g}(q) q^{1 / 2}}{q+1} \lambda_{g}(n)\right)
$$

with the convention $\lambda_{g}(x)=0$ for $x \in \mathbb{Q} \backslash \mathbb{Z}$. Then each $g \in \mathcal{B}_{1}^{*}$ has a Fourier expansion of type (2.5) with $\lambda_{g}^{*}(n)$ in place of $\lambda_{g}(n)$. For $g \in \mathcal{B}_{1}$ and $q \nmid n m$ it follows that

$$
\begin{aligned}
& \lambda_{g}(n) \bar{\lambda}_{g}(m)+\lambda_{g}^{*}(n) \bar{\lambda}_{g}^{*}(m)=c_{1}(g, q) \lambda_{g}(n) \bar{\lambda}_{g}(m), \\
& c_{1}(g, q)=\left(1-\frac{\lambda_{g}(q)^{2} q}{(q+1)^{2}}\right)^{-1} \asymp 1
\end{aligned}
$$

by (2.7) and

$$
\begin{aligned}
& q^{1 / 2}\left(\lambda_{g}(q n) \bar{\lambda}_{g}(m)+\lambda_{g}^{*}(q n) \bar{\lambda}_{g}^{*}(m)\right)=c_{2}(g, q) \lambda_{g}(n) \bar{\lambda}_{g}(m), \\
& c_{2}(g, q)=q^{1 / 2} \lambda_{g}(q)\left(1-\frac{q}{q+1}\left(1-\frac{\lambda_{g}^{2}(q)}{q+1}\right)\left(1-\frac{q \lambda_{g}(q)^{2}}{(q+1)^{2}}\right)^{-1}\right) \\
& \ll q^{-1 / 2}\left|\lambda_{g}(q)\right| \ll 1 .
\end{aligned}
$$

The main point here is that even though for $g \in \mathcal{B}_{1}$ the formula (2.3) does not hold, an appropriate analogue is true if one combines the Fourier coefficients of $g$ and $g_{q}$. 
Similar Fourier expansions hold for the Eisenstein series $E_{\mathfrak{a}}(z, s)$. Let

$$
\eta(n, t):=\sum_{a d=|n|}(a / d)^{i t} .
$$

Then

$$
\begin{aligned}
E_{\mathfrak{a}}(z, 1 / 2+i t)= & \delta_{\mathfrak{a}=\infty} y^{1 / 2+i t}+\phi_{\mathfrak{a}}(1 / 2+i t) y^{1 / 2-i t} \\
& +\rho_{\mathfrak{a}}(1, t) \sum_{n \neq 0} \eta_{\mathfrak{a}}(n, t) \sqrt{y} K_{i t}(2 \pi|n| y) e(n x)
\end{aligned}
$$

where $\phi_{\mathfrak{a}}(s)$ is a meromorphic function that we do not need to specify, and (see [CI, (3.25)])

$$
\begin{aligned}
& \left|\rho_{\mathfrak{a}}(1, t)\right|=\left(\frac{4 \cosh (\pi t)}{q\left|\zeta^{(q)}(1+2 i t)\right|}\right)^{1 / 2}, \\
& \eta_{\infty}(n, t)=\frac{\eta(n, t)}{q^{1 / 2+i t}}-q^{1 / 2} \eta(n / q, t), \quad \eta_{0}(n, t)=\eta(n, t)-q^{-i t} \eta(n / q, t)
\end{aligned}
$$

with the above convention that $\eta(x, t)=0$ for $x \in \mathbb{Q} \backslash \mathbb{Z}$. For $q \nmid m n$ it follows that

$$
\eta_{\infty}(n, t) \eta_{\infty}(m,-t)+\eta_{0}(n, t) \eta_{0}(m,-t)=\left(1+\frac{1}{q}\right) \eta(n, t) \eta(m,-t)
$$

and

$$
q^{1 / 2}\left(\eta_{\infty}(q n, t) \eta_{\infty}(m,-t)+\eta_{0}(q n, t) \eta_{0}(m,-t)\right)=\frac{\eta(q, t)}{q^{1 / 2}} \eta(n, t) \eta(m,-t) .
$$

One of the most important tools is the Kuznetsov formula. Let $n, m \in \mathbb{Z}$ be coprime to $q$ (in particular non-zero), and let $h$ be an even holomorphic function in $|\Im t|<3 / 4$ such that $h(t) \ll(1+|t|)^{-3}$. Then the Kuznetsov formula [IK, p. 409] together with the previous calculations (2.9), (2.13), (2.11) and (2.14) implies that

$$
\begin{aligned}
& 2 \sum_{g \in \mathcal{B}_{1}} \frac{c_{1}(g, q) \lambda_{g}(n) \bar{\lambda}_{g}(m)}{(q+1) L\left(1, \mathrm{Ad}^{2} g\right)} h\left(t_{g}\right)+2 \sum_{g \in \mathcal{B}_{q}} \frac{\lambda_{g}(n) \bar{\lambda}_{g}(m)}{q^{\prime} L\left(1, \mathrm{Ad}^{2} g\right)} h\left(t_{g}\right)+\int_{\mathbb{R}} \frac{\eta(n, t) \eta(m,-t)}{q^{\prime \prime}\left|\zeta^{(q)}(1+2 i t)\right|^{2}} h(t) \frac{d t}{\pi} \\
& \quad=\delta_{n, m} \int_{0}^{\infty} h(t) \frac{d^{*} t}{\pi^{2}}+\sum_{q \mid c} \frac{1}{c} S(n, m, c) \int_{0}^{\infty} \mathcal{J}^{ \pm}\left(\frac{\sqrt{|n m|}}{c}, t\right) h(t) \frac{d^{*} t}{\pi}
\end{aligned}
$$

where $\pm=\operatorname{sgn}(m n), d^{*} t=t \tanh (\pi t) d t, q^{\prime}=q^{2} /(q-1)$ as in (2.10), $q^{\prime \prime}=q^{2} /(q+1)$ and

$$
\mathcal{J}^{ \pm}(x, t)=\left\{\begin{array}{l}
\frac{2 i}{\sinh (\pi t)}\left(J_{2 i t}(4 \pi x)-J_{-2 i t}(4 \pi x)\right), \\
\frac{2 i}{\sinh (\pi t)}\left(I_{2 i t}(4 \pi x)-I_{-2 i t}(4 \pi x)\right)=\frac{4}{\pi} K_{2 i t}(4 \pi x) \cosh (\pi t) .
\end{array}\right.
$$


Similarly, for $q \nmid n m$ we obtain by (2.12) and (2.15) instead of (2.11) and (2.14) that

$$
\begin{aligned}
& 2 \sum_{g \in \mathcal{B}_{1}} \frac{c_{2}(g, q) \lambda_{g}(n) \bar{\lambda}_{g}(m)}{(q+1) L\left(1, \mathrm{Ad}^{2} g\right)} h\left(t_{g}\right)+2 q^{1 / 2} \sum_{g \in \mathcal{B}_{q}} \frac{\lambda_{g}(q n) \bar{\lambda}_{g}(m)}{q^{\prime} L\left(1, \operatorname{Ad}^{2} g\right)} h\left(t_{g}\right)+\int_{\mathbb{R}} \frac{\eta(q n, t) \eta(m,-t)}{q^{3 / 2}\left|\zeta^{(q)}(1+2 i t)\right|^{2}} h(t) \frac{d t}{\pi} \\
&=q^{1 / 2} \sum_{q \mid c} \frac{1}{c} S(q n, m, c) \int_{0}^{\infty} \mathcal{J}^{ \pm}\left(\frac{\sqrt{|q n m|}}{c}, t\right) h(t) \frac{d^{*} t}{\pi} .
\end{aligned}
$$

Let

$$
\mathcal{J}^{0}(x, t):=\frac{1}{2}\left(\mathcal{J}^{+}(x, t)+\mathcal{J}^{-}(x, t)\right)
$$

Adding the Kuznetsov formula for $n m>0$ and $n m<0$, we can single out even Maaß forms:

$$
\begin{aligned}
2 \sum_{\substack{g \in \mathcal{B}_{1} \\
g \text { even }}} \frac{c_{1}(g, q) \lambda_{g}(n) \bar{\lambda}_{g}(m)}{(q+1) L\left(1, \mathrm{Ad}^{2} g\right)} h\left(t_{g}\right)+2 \sum_{\substack{g \in \mathcal{B}_{q} \\
g \text { even }}} \frac{\lambda_{g}(n) \bar{\lambda}_{g}(m)}{q^{\prime} L\left(1, \mathrm{Ad}^{2} g\right)} h\left(t_{g}\right) \\
\quad+\sum_{\mathfrak{a}} \int_{\mathbb{R}} \frac{\eta(n ; t) \eta(m ;-t)}{q^{\prime \prime}\left|\zeta^{(q)}(1+2 i t)\right|^{2}} h(t) \frac{d t}{\pi} \\
=\delta_{n, m} \int_{0}^{\infty} h(t) \frac{d^{*} t}{2 \pi^{2}}+\sum_{q \mid c} \frac{1}{c} S(n, m, c) \int_{0}^{\infty} \mathcal{J}^{0}\left(\frac{\sqrt{n m}}{c}, t\right) h(t) \frac{d^{*} t}{\pi}
\end{aligned}
$$

for $m, n \in \mathbb{N}, q \nmid n m$, as well as

$$
\begin{gathered}
2 \sum_{\substack{g \in \mathcal{B}_{1} \\
g \text { even }}} \frac{c_{2}(g, q) \lambda_{g}(n) \bar{\lambda}_{g}(m)}{(q+1) L\left(1, \mathrm{Ad}^{2} g\right)} h\left(t_{g}\right)+2 q^{1 / 2} \sum_{\substack{g \in \mathcal{B}_{q} \\
g \text { even }}} \frac{\lambda_{g}(q n) \bar{\lambda}_{g}(m)}{q^{\prime} L\left(1, \mathrm{Ad}^{2} g\right)} h\left(t_{g}\right) \\
\quad+\int_{\mathbb{R}} \frac{\eta(q n, t) \eta(m,-t)}{q^{3 / 2}\left|\zeta^{(q)}(1+2 i t)\right|^{2}} h(t) \frac{d t}{\pi} \\
=q^{1 / 2} \sum_{q \mid c} \frac{1}{c} S(q n, m, c) \int_{0}^{\infty} \mathcal{J}^{ \pm}\left(\frac{\sqrt{q n m}}{c}, t\right) h(t) \frac{d^{*} t}{\pi} .
\end{gathered}
$$

We will need all four versions (2.16)-(2.19) in Section 5.

\section{Triple product $L$-functions}

Let $f, g \in \mathcal{B}_{q}$. Then we can define the triple product $L$-function

$$
L(s, f \times \bar{f} \times g)=L\left(s, \operatorname{Ad}^{2} f \times g\right) L(s, g) .
$$


The local factors, root number and conductor have been computed in [Wa, Section 3.1]. Let $\Gamma_{\mathbb{R}}(s):=\Gamma(s / 2) \pi^{-s / 2}$. Then

$$
\begin{aligned}
L_{\infty}(s, g) & :=\prod_{ \pm} \Gamma_{\mathbb{R}}\left(s \pm i t_{g}\right), \\
\Lambda(s, g) & =L(s, g) L_{\infty}(s, g)=(-1)^{\delta_{g}}\left(-\lambda_{g}(q) q^{1 / 2}\right) q^{1 / 2-s} \Lambda(1-s, g) .
\end{aligned}
$$

Similarly,

$$
\begin{aligned}
L_{\infty}\left(s, \operatorname{Ad}^{2} f \times g\right) & =\prod_{ \pm} \prod_{\nu=-1}^{1} \Gamma_{\mathbb{R}}\left(s+2 i v t_{f} \pm i t_{g}\right), \\
\Lambda\left(s, \operatorname{Ad}^{2} f \times g\right) & =L\left(s, \operatorname{Ad}^{2} f \times g\right) L_{\infty}\left(s, \operatorname{Ad}^{2} f \times g\right) \\
& =(-1)^{\delta_{g}}\left(q^{4}\right)^{1 / 2-s} \Lambda\left(1-s, \operatorname{Ad}^{2} f \times g\right) .
\end{aligned}
$$

By [LR, Theorem 1], the central value $L(1 / 2, g)$ is non-negative. Moreover, by [JS] the self-dual lift $\operatorname{Ad}^{2} f\left(=\operatorname{sym}^{2} f\right)$ is orthogonal (its symmetric square $L$-function has a pole at $s=1)$, hence by [La, Theorem 1.1], the central value $\Lambda\left(1 / 2, \operatorname{Ad}^{2} f \times g\right)$ is nonnegative too, and an inspection of the archimedean $L$-factors shows that the same holds for $L\left(1 / 2, \operatorname{Ad}^{2} f \times g\right)$, hence also for $L(1 / 2, f \times \bar{f} \times g)$. We note that $L\left(s, \operatorname{Ad}^{2} f \times g\right)$ and hence $L(s, f \times \bar{f} \times g)$ vanishes at $s=1 / 2$ if $g$ is odd.

The adjoint square lift of $f$ is a self-dual automorphic form on $\mathrm{GL}_{3}$ with Fourier coefficients $A(m, k)$ satisfying $A(m, 1)=\sum_{a b^{2}=m} \lambda_{f}\left(a^{2}\right)$ for $q \nmid m$. Using Hecke relations, we can express all $A(m, k)$ in terms of the Hecke eigenvalues of $\lambda_{f}$ as follows: by Möbius inversion and [Go, Theorem 6.4.11] we have

$$
A(m, k)=\sum_{d \mid(m, k)} \mu(d) A\left(\frac{m}{d}, 1\right) A\left(1, \frac{k}{d}\right)
$$

whenever $q \nmid m k$. Hence

$$
L^{(q)}\left(s, \operatorname{Ad}^{2} f \times g\right)=\sum_{q \nmid m k} \frac{A(m, k) \lambda_{g}(m)}{m^{s} k^{2 s}}=\sum_{q \nmid d a b m k} \frac{\mu(d) \lambda_{f}\left(m^{2}\right) \lambda_{f}\left(k^{2}\right) \lambda_{g}\left(d m a^{2}\right)}{m^{s} a^{2 s} k^{2 s} b^{4 s} d^{3 s}} .
$$

Using the explicit shape of the Euler factor at $q$ (see [Wa]), we find

$$
\begin{aligned}
L\left(s, \operatorname{Ad}^{2} f \times g\right) & =\left(1-\frac{\lambda g(q)}{q^{s}}\right)^{-1}\left(1-\frac{\lambda g(q)}{q^{s+1}}\right)^{-1} L^{(q)}\left(s, \operatorname{Ad}^{2} f \times g\right) \\
& =: \sum_{m} \frac{\lambda \operatorname{Ad}^{2} f \times g}{m^{s}}(m)
\end{aligned}
$$

say. Note that by (2.3) the coefficients divisible by $q$ are small. Since these are purely formal computations with local Euler factors, (3.1) holds also for $f$ and/or $g$ in $\mathcal{B}_{1}$, and 
analogous formulas hold for Eisenstein series:

$$
\begin{aligned}
\left|L^{(q)}\left(s+i t, \mathrm{Ad}^{2} f\right)\right|^{2} & =\sum_{q \nmid d a b n m} \frac{\mu(d) \lambda_{f}\left(m^{2}\right) \lambda_{f}\left(k^{2}\right) \eta\left(d m a^{2}, t\right)}{m^{s} a^{2 s} k^{2 s} b^{4 s} d^{3 s}}, \\
\left|L^{(q)}(s+i t, g)\right|^{2} L^{(q)}(s, g) & =\sum_{q \nmid d a b m k} \frac{\mu(d) \eta\left(m^{2}, t\right) \eta\left(k^{2}, t\right) \lambda_{g}\left(d m a^{2}\right)}{m^{s} a^{2 s} k^{2 s} b^{4 s} d^{3 s}} .
\end{aligned}
$$

We have already seen that $L(s, f \times \bar{f} \times g)$ has conductor $q^{5}$ for $f, g \in \mathcal{B}_{q}$. If one of the factors has level one or is an Eisenstein series, the conductor drops; more precisely, all the $L$-functions

$$
\begin{array}{lll}
L(s, f \times \bar{f} \times g), & |L(s+i t, f \times \bar{f})|^{2}, & f \in \mathcal{B}_{q}, g \in \mathcal{B}_{1}, \\
L(s, f \times \bar{f} \times g), & |L(s+i t, g)|^{2} L(s, g)^{2}, & f \in \mathcal{B}_{1}, g \in \mathcal{B}_{q},
\end{array}
$$

have conductor $q^{4}$. We will use this observation in Sections 5 and 6 .

It is a deep result [KSh] that $\mathrm{Ad}^{2} f \times g$ corresponds to an automorphic form on $\mathrm{GL}_{6}$. Hence the Rankin-Selberg $L$-function $L\left(s,\left(\operatorname{Ad}^{2} f \times g\right) \times\left(\operatorname{Ad}^{2} f \times g\right)\right)$ has the properties of [Li, Theorem 2], and we have the upper bound

$$
\sum_{m \leq x}\left|\lambda_{\operatorname{Ad}^{2} f \times g}(m)\right|^{2} \ll x\left(q\left(1+\left|t_{g}\right|+\left|t_{f}\right|\right) x\right)^{\varepsilon} .
$$

We need a somewhat sophisticated and carefully designed approximate functional equation and borrow some ideas from [B12]. Let $A_{1}, A_{2} \geq 10$ be integers and define

$$
G(u)=\left(\cos \frac{\pi u}{4 A_{1}}\right)^{-100 A_{1}}, \quad G_{1}(u, t):=\prod_{ \pm} \prod_{ \pm} \prod_{\ell=0}^{A_{2}}\left(\frac{1 / 2 \pm u \pm i t}{2}+\ell\right)
$$

and

$$
G_{2}\left(u, t_{1}, t_{2}\right):=\prod_{ \pm} \prod_{ \pm} \prod_{\nu=-1}^{1} \prod_{\ell=0}^{3 A_{2}}\left(\frac{1 / 2 \pm u \pm i t_{1}+2 i v t_{2}}{2}+\ell\right) .
$$

Clearly $G_{1}$ and $G_{2}$ are holomorphic and even in all variables, and $G$ is even and holomorphic in $|\Re u|<2 A_{1}$. Moreover, for $t, t_{1}, t_{2} \in \mathcal{T}$ we have

$$
G_{1}(0, t), G_{2}\left(0, t_{1}, t_{2}\right) \gg 1 .
$$

For this lower bound we either need that $\left|\Im t_{1}\right|,\left|\Im t_{2}\right| \leq 1 / 6-\delta$, or any non-trivial bound towards the Ramanujan conjecture for the infinite place of the $\mathrm{GL}_{6}$ automorphic form $\operatorname{Ad}^{2} f \times g$. Both results are known [KS, LRS]. Let

$$
V_{1}(y ; t)=\frac{1}{2 \pi i} \int_{(2)} G(u) G_{1}(u, t) \prod_{ \pm} \frac{\Gamma_{\mathbb{R}}(1 / 2+u \pm i t)}{\Gamma_{\mathbb{R}}(1 / 2 \pm i t)} y^{-u} \frac{d u}{u}
$$


and

$$
V_{2}\left(y ; t_{1}, t_{2}\right)=\frac{1}{2 \pi i} \int_{(2)} G(u) G_{2}\left(u, t_{1}, t_{2}\right) \prod_{ \pm} \prod_{\nu=-1}^{1} \frac{\Gamma_{\mathbb{R}}\left(1 / 2+u \pm i t_{1} \pm 2 i v t_{2}\right)}{\Gamma_{\mathbb{R}}\left(1 / 2 \pm i t_{1} \pm 2 i v t_{2}\right)} y^{-u} \frac{d u}{u}
$$

The weight functions $V_{1}, V_{2}$ have the following properties:

Lemma 1. (a) The function $V_{1}(y ; t)$ is smooth for $y>0$ and holomorphic in $|\Im t| \leq 2 A_{2}$ and satisfies the uniform bound

$y^{j} V_{1}^{(j)}(y ; t) \ll(1+|t|)^{4\left(A_{2}+1\right)}\left(1+\frac{y}{1+|t|}\right)^{-A_{1}} \ll(1+|t|)^{A_{1}+4\left(A_{2}+1\right)}(1+y)^{-A_{1}}$

in this region for fixed $j \in \mathbb{N}_{0}$. Its Mellin transform with respect to the first variable, $\widehat{V}_{1}(u ; t)$, is holomorphic in $\Re u \geq \varepsilon$ whenever $t \in \mathcal{T}$. In this region it satisfies the uniform bound

$$
\widehat{V}_{1}(u ; t) \ll \Re u, \varepsilon e^{-\Im|u|}(1+|t|)^{4\left(A_{2}+1\right)+\Re u} .
$$

Moreover,

$$
\widehat{V}_{1}(1 / 2 \pm i t, t)=0 \text {. }
$$

(b) The function $V_{2}\left(y, t_{1}, t_{2}\right)$ is smooth in $y>0$ and holomorphic in $\left|\Im t_{1}\right|,\left|\Im t_{2}\right| \leq 2 A_{2}$ and satisfies the uniform bound

$$
\begin{aligned}
y^{j} V_{2}^{(j)}(y ; t) & \ll\left(1+\left|t_{1}\right|+\left|t_{2}\right|\right)^{12\left(3 A_{2}+1\right)}\left(1+\frac{y}{\left(1+\left|t_{1}\right|+|t|_{2}\right)^{3}}\right)^{-A_{1}} \\
& \ll\left(1+\left|t_{1}\right|+\left|t_{2}\right|\right)^{3 A_{1}+12\left(3 A_{2}+1\right)}(1+y)^{-A_{1}}
\end{aligned}
$$

in this region for fixed $j \in \mathbb{N}_{0}$. Its Mellin transform with respect to the first variable, $\widehat{V}_{2}\left(u ; t_{1}, t_{2}\right)$, is holomorphic in $\Re u \geq \varepsilon$ whenever $t_{1}, t_{2} \in \mathcal{T}$. In this region it satisfies the uniform bound

$$
\widehat{V}_{2}\left(u ; t_{1}, t_{2}\right) \ll \Re u, \varepsilon e^{-\Im|u|}\left(1+\left|t_{1}\right|+\left|t_{2}\right|\right)^{12\left(3 A_{2}+1\right)+3 \Re u} .
$$

Proof. This follows easily from the definition of $G, G_{1}, G_{2}$ with appropriate contour shifts.

Let $g \in \mathcal{B}_{q}$ be even. Then the usual technique (e.g. [IK, p. 98] or [B12, Section 2]) shows

$$
G_{1}\left(0, t_{g}\right) L(1 / 2, g)=\left(1-\lambda_{g}(q) q^{1 / 2}\right) \sum_{n} \frac{\lambda_{g}(n)}{n^{1 / 2}} V_{1}\left(\frac{n}{q^{1 / 2}} ; t_{g}\right)
$$

and

$$
G_{2}\left(0, t_{g}, t_{f}\right) L\left(1 / 2, \operatorname{Ad}^{2} f \times g\right)=2 \sum_{m} \frac{\lambda \mathrm{Ad}^{2} f \times g}{m^{1 / 2}} V_{2}\left(\frac{m}{q^{2}} ; t_{g}, t_{f}\right) .
$$




\section{Character sums}

For future reference we state some useful results for character sums. We quote from [B11, Section 3]. For a positive or negative discriminant $D$ of a quadratic number field let $\chi_{D}=$ $\left(\frac{D}{-}\right)$ be the associated Dirichlet character. Define $\epsilon_{c}=1$ if $c>0$ and $\epsilon_{c}=i$ if $c<0$. If $c=c_{1} c_{2}^{2}$ is odd and positive with $\mu\left(c_{1}\right)^{2}=1$ let $c^{*}:=\chi_{-4}\left(c_{1}\right) c_{1}$. We need to evaluate the sum

$$
G(d, h ; q):=\sum_{x(q)} e\left(\frac{d x^{2}+h x}{q}\right)
$$

for integers $d \in \mathbb{Z} \backslash\{0\}, h \in \mathbb{Z}, q \in \mathbb{N}$. Clearly

$$
G(d, h ; q)=\delta_{(d, q) \mid h} G(d /(d, q), h /(d, q) ; q /(d, q)),
$$

so it suffices to compute the sum for $(d, q)=1$. We write $q=s 2^{\alpha}$ with $s$ odd. Then we have ([B11, Lemma 2])

$$
\begin{aligned}
& G(d, h ; q) \\
& \quad= \begin{cases}\sqrt{q} \epsilon_{q^{*}} \chi_{q^{*}}(d) e\left(\frac{-\overline{4 d} h^{2}}{q}\right), & \alpha=0, \\
\sqrt{2 q} \epsilon_{s^{*}} \chi_{s^{*}}(2 d) e\left(\frac{-\overline{8 d} h^{2}}{s}\right), & \alpha=1, h \text { odd }, \\
\sqrt{q} \epsilon_{s^{*}} \chi_{s^{*}}(d) e\left(\frac{-\bar{d}\left(h^{\prime}\right)^{2}}{q}\right)\left(1+i \chi_{-4}(s d)\right), & \alpha \geq 2 \text { even, } h=2 h^{\prime} \text { even, } \\
\sqrt{q} \epsilon_{s^{*}} \chi_{8 s^{*}}(d) e\left(\frac{-\bar{d}\left(h^{\prime}\right)^{2}}{q}\right)\left(1+i \chi_{-4}(s d)\right), & \alpha \geq 3 \text { odd, } h=2 h^{\prime} \text { even, } \\
0, & \text { otherwise, }\end{cases}
\end{aligned}
$$

whenever $(d, q)=1$. If $\psi$ is a real character of conductor $s, q=s s_{1} s_{2}$ with $s_{1} \mid s^{\infty}$ and $\left(s, s_{2}\right)=1$, and $\Delta \in \mathbb{Z}$, then we have $([\mathrm{B} 11,(3.2)])$

$$
\left|\sum_{d(q)}^{*} \psi(d) e\left(\frac{d \Delta}{q}\right)\right|=\left|\delta_{s_{1} \mid \Delta} \psi\left(s_{2} \frac{\Delta}{s_{1}}\right) s_{1} r_{s_{2}}(\Delta) \sqrt{s} \epsilon_{s^{*}}\right| \leq \sqrt{q s_{1} s_{2}}
$$

where $r_{q}(\Delta)$ is the Ramanujan sum.

For a Schwartz class function $W$ we denote by $\check{W}$ its Fourier transform.

Lemma 2. Let $\gamma \in \mathbb{N}, \alpha \in \mathbb{Z}$, and let $W$ be a Schwartz class function. Then

$$
\sum_{n \in \mathbb{Z}} S(n, \alpha, \gamma) W(n)=\sum_{\substack{h_{1} \in \mathbb{Z} \\\left(h_{1}, \gamma\right)=1}} e\left(-\frac{\alpha \bar{h}_{1}}{\gamma}\right) \check{W}\left(\frac{h_{1}}{\gamma}\right) .
$$

Proof. This is a direct consequence of the Poisson summation formula. 
Lemma 3. Let $c, r \in \mathbb{N}, \beta, \kappa, h \in \mathbb{Z}$. Write $r=r_{1} r_{2}^{2}$ with $\mu\left(r_{1}\right)^{2}=1$ and write $f=$ $(c, r), c=f \tilde{c}, r=f \tilde{r}$ with $(\tilde{r}, \tilde{c})=1$. Then

$$
\left|\sum_{m(q \tilde{c} \tilde{r} f)} e\left(\frac{m \beta}{q c}\right) S\left(m^{2}, \kappa, q r\right) e\left(\frac{m h}{q \tilde{c} \tilde{r} f}\right)\right| \begin{cases}\leq 2 q r r_{2} \tilde{c} & \text { if } \beta \tilde{r} \equiv-h(\tilde{c}), \\ =0 & \text { otherwise. }\end{cases}
$$

Proof. Opening the Kloosterman sum, the exponential sum in question equals

$$
\sum_{x(q r)}^{*} e\left(\frac{\kappa \bar{x}}{q r}\right) G(x \tilde{c}, h+\beta \tilde{r}, q \tilde{c} \tilde{r} f)=\tilde{c} \delta_{\tilde{c} \mid h+\beta \tilde{r}} \sum_{x(q r)}^{*} e\left(\frac{\kappa \bar{x}}{q r}\right) G(x,(h+\beta \tilde{r}) / \tilde{c}, q r) .
$$

For notational simplicity let us write $\gamma:=(h+\beta \tilde{r}) / \tilde{c}$. We evaluate the Gauß sum using (4.1). To this end, we write $q r=s 2^{a}$ with $s$ odd and also recall $r=r_{1} r_{2}^{2}$ with $\mu\left(r_{1}\right)^{2}=1$. We distinguish several very similar cases. If $a=0$, we obtain

$$
\sqrt{q r} \epsilon_{(q r) * \tilde{c}} \delta_{\tilde{c} \mid h+\beta \tilde{r}} \sum_{x(q r)}^{*} e\left(\frac{\kappa \bar{x}}{q r}\right) \chi_{(q r) *(x) e}\left(\frac{-\overline{4 x} \gamma^{2}}{q r}\right),
$$

and the desired bound (without the factor 2) follows directly from (4.2). If $a=1$, we obtain

$$
\sqrt{2 q r} \epsilon_{s^{*}} \tilde{c} \delta_{\tilde{c} \mid h+\beta \tilde{r}} \delta_{2 \nmid \gamma} \sum_{x(q r)}^{*} e\left(\frac{\kappa \bar{x}}{q r}\right) \chi_{s^{*}}(2 x) e\left(\frac{-\overline{8 x} \gamma^{2}}{s}\right) .
$$

The $x$-sum equals in absolute value

$$
\left|\sum_{x(s)}^{*} e\left(\frac{\kappa \overline{2 x}}{s}\right) \chi_{s^{*}}(2 x) e\left(\frac{-\overline{8 x} \gamma^{2}}{s}\right)\right| \leq \sqrt{s} r_{2}=r_{2} \sqrt{q r / 2},
$$

and the lemma follows again (without the factor 2). If $a \geq 2$ is even, we have

$$
\sqrt{q r} \epsilon_{s^{*}} \tilde{c} \delta_{\tilde{c} \mid h+\beta \tilde{r}} \delta_{2 \mid \gamma} \sum_{x(q r)}^{*} e\left(\frac{\kappa \bar{x}}{q r}\right) \chi_{s^{*}}(x) e\left(\frac{-\bar{x}(\gamma / 2)^{2}}{q r}\right)\left(1+i \chi_{-4}(s x)\right),
$$

and the lemma follows from (4.2). The case $a \geq 3$ odd is identical.

Lemma 4. Let c, $r \in \mathbb{N}$ and let $\beta, \kappa \in \mathbb{Z}$. Write $r=r_{1} r_{2}^{2}$ with $\mu\left(r_{1}\right)^{2}=1$ and write $f=(c, r), c=f \tilde{c}, r=f \tilde{r}$ with $(\tilde{r}, \tilde{c})=1$. Let $W$ be a Schwartz class function. Then

$$
\sum_{m \in \mathbb{Z}} e\left(\frac{m \beta}{q c}\right) S\left(m^{2}, \kappa, q r\right) W(m) \ll r_{2} \sum_{\substack{h_{2} \in \mathbb{Z} \\ h_{2} \equiv-\beta \tilde{r}(\tilde{c})}}\left|\check{W}\left(\frac{h_{2}}{q \tilde{c} \tilde{r} f}\right)\right| .
$$

Proof. This is a direct consequence of the Poisson summation formula and Lemma 3.

Finally we recall Weil's bound for Kloosterman sums,

$$
|S(a, b, c)| \leq(a, b, c)^{1 / 2} c^{1 / 2} \tau(c) .
$$

By twisted multiplicativity we see

$$
S(q a, b, q c)=-S(a, b \bar{q}, c)
$$

whenever $q \nmid b c$. 


\section{The main transformation}

In this section we use Watson's formula and the Kuznetsov formula to transform the quantity of interest, $\sum_{f}\|f\|_{4}^{4}$, into character sums.

Let $f \in \mathcal{B}_{q}$ be an $L^{2}$-normalized cuspidal Hecke-Maaß newform of level $q$ with spectral parameter $t_{f} \leq T$. We begin with Parseval's identity

$$
\begin{aligned}
\|f\|_{4}^{4}=\left\langle|f|^{2},|f|^{2}\right\rangle= & V(q)^{-1}\left|\left\langle|f|^{2}, 1\right\rangle\right|^{2} \\
& +\sum_{g \in \mathcal{B}}\left|\left\langle|f|^{2}, g\right\rangle\right|^{2}+\sum_{\mathfrak{a}} \int_{\mathbb{R}}\left|\left\langle|f|^{2}, E_{\mathfrak{a}}(\cdot, 1 / 2+i t)\right\rangle\right|^{2} \frac{d t}{4 \pi} .
\end{aligned}
$$

We study the various terms on the right hand side.

The constant function contributes $V(q)^{-1}=O(1 / q)$.

Since the Laplace operator is symmetric, we find

$$
\begin{aligned}
\left|\left\langle|f|^{2}, g\right\rangle\right| & =\left|\left(1 / 4+t_{g}^{2}\right)^{-a}\left\langle\Delta^{a}|f|^{2}, g\right\rangle\right| \leq\left|1 / 4+t_{g}^{2}\right|^{-a}\left\|\Delta^{a}|f|^{2}\right\|_{2} \\
& \ll q\left|1 / 4+t_{g}^{2}\right|^{-a}\left\|\Delta^{a}|f|^{2}\right\|_{\infty}
\end{aligned}
$$

for any $a \in \mathbb{N}$. We recall that $|f|^{2}$ is invariant under the group $\Gamma_{0}^{*}(q)$, generated by $\Gamma_{0}(q)$ and the Fricke involution, and a fundamental domain for $\Gamma_{0}^{*}(q)$ is contained in the Siegel set $\{z \in \mathbb{H}|y \geq 1 /(2 q)| x \mid, \leq 1 / 2\}$. Since $\Delta$ is $\mathrm{SL}_{2}(\mathbb{R})$-invariant, we conclude that

$$
\left\|\Delta^{a}|f|^{2}\right\|_{\infty}=\left.\sup _{y \geq 1 /(2 q)}\left|\Delta^{a}\right| f\right|^{2}(z) \mid .
$$

Inserting the Fourier expansion (2.5), we have

$$
\Delta^{a}|f|^{2}(z)=\left|\rho_{f}(1)\right|^{2} \sum_{n, m \neq 0} \lambda_{f}(n) \overline{\lambda_{f}(m)} \Delta^{a}\left(y K_{i t_{f}}(2 \pi|n| y) K_{i t_{f}}(2 \pi|m| y) e((n-m) x)\right) .
$$

Since

$$
\frac{d^{a}}{d y^{a}} \cosh (\pi t / 2) K_{i t}(y) \ll t, a e^{-y}\left(1+y^{-a-|\Im t|}|\log y|\right)
$$

for $t \in \mathcal{T}$, we conclude from (2.9) and (2.1) that

$$
\left|\rho_{f}(1)\right|^{2} \Delta^{a}\left(y K_{i t_{f}}(2 \pi|n| y) K_{i t_{f}}(2 \pi|m| y) e((n-m) x)\right) \ll_{a, t_{f}} \frac{e^{-(|n|+|m|) y}}{q|n m|^{1 / 2}},
$$

which together with (2.6) implies $\left\|\Delta^{a}|f|^{2}\right\|_{\infty} \ll_{a, T, \varepsilon} q^{\varepsilon}$. In particular, the oldforms contribute

$$
\begin{aligned}
\sum_{g \in \mathcal{B} \backslash \mathcal{B}_{q}}\left|\left\langle|f|^{2}, g\right\rangle\right|^{2} & =\sum_{\substack{g \in \mathcal{B} \backslash \mathcal{B}_{q} \\
t_{g} \leq q^{\varepsilon}}}\left|\left\langle|f|^{2}, g\right\rangle\right|^{2}+\sum_{\substack{g \in \mathcal{B} \backslash \mathcal{B}_{q} \\
t_{g}>q^{\varepsilon}}}\left|\left\langle|f|^{2}, g\right\rangle\right|^{2} \\
& \ll_{\varepsilon, T} \sum_{\substack{g \in \mathcal{B} \backslash \mathcal{B}_{q} \\
t_{g} \leq q^{\varepsilon}}}\|g\|_{\infty}^{2}+q^{1+\varepsilon} \sum_{\substack{g \in \mathcal{B} \backslash \mathcal{B}_{q} \\
t_{g}>q^{\varepsilon}}}\left(1+\left|t_{g}\right|\right)^{-3-2 / \varepsilon} \ll q^{\varepsilon-1}
\end{aligned}
$$

by (2.4) and (2.2). 
By Watson's formula [Wa, Theorem 4.1] and positivity, the newforms contribute

$$
\begin{aligned}
& \ll \frac{1}{q^{2}} \sum_{\substack{g \in \mathcal{B}_{q} \\
g \text { even }}} \frac{\Lambda(1 / 2, f \times \bar{f} \times g)}{\Lambda\left(1, \operatorname{Ad}^{2} f\right)^{2} \Lambda\left(1, \mathrm{Ad}^{2} g\right)} \\
& \ll_{\varepsilon, T} \frac{1}{q^{2-\varepsilon}} \sum_{\substack{g \in \mathcal{B}_{q} \\
g \text { even }}} \frac{L(1 / 2, f \times \bar{f} \times g)}{L\left(1, \operatorname{Ad}^{2} f\right) L\left(1, \operatorname{Ad}^{2} g\right)} e^{-\frac{3}{2} \pi\left|t_{g}\right|} .
\end{aligned}
$$

Here we used a lower bound [HL] on $L\left(1, \operatorname{Ad}^{2} f\right)$. Since $f$ is an eigenfunction of the Fricke involution which is the scaling matrix for the cusp $\mathfrak{a}=0$, the contribution of the two cusps is the same. By the unfolding technique we find, as in (2.8),

$$
\left\langle|f|^{2}, E_{\infty}(\cdot, s)\right\rangle=\left|\rho_{f}(1)\right|^{2} \frac{2 L(s, f \times \bar{f})}{\zeta(2 s)} \frac{\Gamma_{\mathbb{R}}(s) \Gamma_{\mathbb{R}}\left(s-2 i t_{f}\right) \Gamma_{\mathbb{R}}\left(s+2 i t_{f}\right)}{2^{2+s} \Gamma_{\mathbb{R}}(1+s)} .
$$

From (2.9) we conclude that the Eisenstein contribution is

$$
\ll_{\varepsilon, T} \frac{1}{q^{2-\varepsilon}} \int_{\mathbb{R}} \frac{|L(1 / 2+i t, f \times \bar{f})|^{2}}{|\zeta(1+2 i t)|^{2}} e^{-\frac{3}{2} \pi|t|} d t \ll q^{\varepsilon-1}
$$

by the convexity bound for $L(1 / 2+i t, f \times \bar{f})$. Combining the above estimates we find

$$
\|f\|_{4}^{4} \ll \frac{1}{q^{2-\varepsilon} L\left(1, \operatorname{Ad}^{2} f\right)} \sum_{\substack{g \in \mathcal{B}_{q} \\ g \text { even }}} \frac{L(1 / 2, f \times \bar{f} \times g)}{L\left(1, \operatorname{Ad}^{2} g\right)} e^{-\frac{3}{2} \pi\left|t_{g}\right|}+q^{\varepsilon-1} .
$$

We insert artificially the factor $G_{1}\left(0, t_{g}\right) G_{2}\left(0, t_{g}, t_{f}\right)$ by positivity and (3.4) and also change the weight function $e^{-(3 / 2) \pi|t|}$ to the function

$$
h(t):=\cosh \left(\frac{t}{2 A_{2}}\right)^{-3 \pi A_{2}} \prod_{\nu=0}^{A_{2}}\left(t^{2}+\left(\frac{1}{2}+v\right)^{2}\right)
$$

Note that this function is holomorphic in $|\Im t|<\pi A_{2}$ and has zeros at the zeros of $\cosh (\pi t)$ in this region. Moreover, $h(t) \gg \exp \left(-\frac{3}{2} \pi|t|\right)$ for $t \in \mathcal{T}$. Using (3.6)-(3.7), we write

$$
\|f\|_{4}^{4} \ll \frac{q^{\varepsilon}}{q^{\prime} L\left(1, \operatorname{Ad}^{2} f\right)}\left(1+\sum_{\substack{g \in \mathcal{B}_{q} \\ g \text { even }}} \frac{2 h\left(t_{g}\right)}{q^{\prime} L\left(1, \operatorname{Ad}^{2} g\right)}\left(1-\lambda_{g}(q) q^{1 / 2}\right) S\right)
$$

where

$$
S=\sum_{n, m} \frac{\lambda_{g}(n) \lambda_{\mathrm{Ad}^{2} f \times g}(m)}{(n m)^{1 / 2}} V_{1}\left(\frac{n}{q^{1 / 2}} ; t_{g}\right) V_{2}\left(\frac{m}{q^{2}} ; t_{g}, t_{f}\right)
$$

and $q^{\prime}$ was defined in (2.10). Hence by (2.2) and (2.4), 


$$
\begin{aligned}
\sum_{t_{f} \leq T}\|f\|_{4}^{4} & \ll_{T, \varepsilon} q^{\varepsilon}+q^{\varepsilon} \sum_{f \in \mathcal{B}_{q}} \frac{2 h\left(t_{f}\right)}{q^{\prime} L\left(1, \mathrm{Ad}^{2} f\right)} \sum_{\substack{g \in \mathcal{B}_{q} \\
g \text { even }}} \frac{2 h\left(t_{g}\right)}{q^{\prime} L\left(1, \mathrm{Ad}^{2} g\right)}\left(1-\lambda_{g}(q) q^{1 / 2}\right) S .
\end{aligned}
$$

(Here we used (2.4) and $L\left(1, \operatorname{Ad}^{2} f\right) \gg_{T} q^{-\varepsilon}$ for the first term on the right hand side.) It is convenient to remove the terms with $q \mid n m$ in $S$. By the rapid decay of $V_{1}$ the terms $q \mid n$ are negligible. Combining (2.3) and (3.2) with (2.4), (2.6), (3.3) and the rapid decay of $V_{2}$, we see by trivial estimates that the contribution of the terms $q \mid m$ in $S$ contributes at most $O\left(q^{\varepsilon-1 / 4}\right)$ to (5.1). Hence by (3.2) we are left with estimating

$$
\Sigma(q, q):=\Sigma_{1}(q, q)-\Sigma_{2}(q, q),
$$

say, where

$$
\begin{aligned}
\Sigma_{1}(q, q):= & \sum_{f \in \mathcal{B}_{q}} \frac{2 h\left(t_{f}\right)}{q^{\prime} L\left(1, \mathrm{Ad}^{2} f\right)} \sum_{\substack{g \in \mathcal{B}_{q} \\
g \text { even }}} \frac{2 h\left(t_{g}\right)}{q^{\prime} L\left(1, \mathrm{Ad}^{2} g\right)} \sum_{q \nmid a b d k} \frac{\mu(d)}{a b^{2} k d^{3 / 2}} \\
& \times \sum_{q \nmid n m} \frac{\lambda_{f}\left(k^{2}\right) \lambda_{g}(n) \lambda_{f}\left(m^{2}\right) \lambda_{g}\left(a^{2} d m\right)}{(n m)^{1 / 2}} V_{1}\left(\frac{n}{q^{1 / 2}} ; t_{g}\right) V_{2}\left(\frac{a^{2} b^{4} k^{2} d^{3} m}{q^{2}} ; t_{g}, t_{f}\right)
\end{aligned}
$$

and

$$
\begin{aligned}
\Sigma_{2}(q, q) & :=q^{1 / 2} \sum_{f \in \mathcal{B}_{q}} \frac{2 h\left(t_{f}\right)}{q^{\prime} L\left(1, \mathrm{Ad}^{2} f\right)} \sum_{\substack{g \in \mathcal{B}_{q} \\
g \text { even }}} \frac{2 h\left(t_{g}\right)}{q^{\prime} L\left(1, \mathrm{Ad}^{2} g\right)} \sum_{q \nmid a b d k} \frac{\mu(d)}{a b^{2} k d^{3 / 2}} \\
& \times \sum_{q \nmid n m} \frac{\lambda_{f}\left(k^{2}\right) \lambda_{g}(q n) \lambda_{f}\left(m^{2}\right) \lambda_{g}\left(a^{2} d m\right)}{(n m)^{1 / 2}} V_{1}\left(\frac{n}{q^{1 / 2}} ; t_{g}\right) V_{2}\left(\frac{a^{2} b^{4} k^{2} d^{3} m}{q^{2}} ; t_{g}, t_{f}\right) .
\end{aligned}
$$

We would like to apply the Kuznetsov formula to the spectral sums over $f$ and $g$. More precisely, we use (2.16) for the sum over $f$ and (2.18) for the sum over even Maaß forms $g$; in $\Sigma_{2}$ we use (2.17) for the $f$-sum and (2.19) for the $g$-sum. However, this requires some preparation, as the $f$ - and $g$-sums run only over cuspidal newforms, and both the oldforms of level 1 as well as the Eisenstein series are missing. Therefore we artificially add and subtract the missing terms and define, in analogy with $\Sigma(q, q)=$ $\Sigma_{1}(q, q)-\Sigma_{2}(q, q)$, eight other quantities $\Sigma(*, *)=\Sigma_{1}(*, *)-\Sigma_{2}(*, *)$ where $* \epsilon$ $\{q, 1, \mathcal{E}\}$ in an obvious way in order to complete the spectral side of the Kuznetsov formula for the $f$ - and $g$-sum respectively. For instance, we write

$$
\begin{aligned}
\Sigma_{1}(1, \mathcal{E}):= & \sum_{f \in \mathcal{B}_{1}} \frac{2 h\left(t_{f}\right)}{(q+1) L\left(1, \mathrm{Ad}^{2} f\right)} \int_{\mathbb{R}} \frac{h(t)}{q^{\prime \prime}\left|\zeta^{(q)}(1+2 i t)\right|^{2}} \sum_{q \nmid a b d k} \frac{\mu(d)}{a b^{2} k d^{3 / 2}} \\
& \times \sum_{q \nmid n m} \frac{c_{1}(f, q) \lambda_{f}\left(k^{2}\right) \eta(n, t) \lambda_{f}\left(m^{2}\right) \eta\left(a^{2} d m,-t\right)}{(n m)^{1 / 2}} \\
& \quad \times V_{1}\left(\frac{n}{q^{1 / 2}} ; t\right) V_{2}\left(\frac{a^{2} b^{4} k^{2} d^{3} m}{q^{2}} ; t, t_{f}\right) \frac{d t}{\pi},
\end{aligned}
$$


and

$$
\begin{aligned}
\Sigma_{2}(1, \mathcal{E}):= & \sum_{f \in \mathcal{B}_{1}} \frac{2 h\left(t_{f}\right)}{(q+1) L\left(1, \mathrm{Ad}^{2} f\right)} \int_{\mathbb{R}} \frac{h(t)}{q^{3 / 2}\left|\zeta^{(q)}(1+2 i t)\right|^{2}} \sum_{q \nmid a b d k} \frac{\mu(d)}{a b^{2} k d^{3 / 2}} \\
& \times \sum_{q \nmid n m} \frac{c_{2}(f, q) \lambda_{f}\left(k^{2}\right) \eta(n,-t) \lambda_{f}^{*}\left(m^{2}\right) \eta\left(a^{2} d m,-t\right)}{(n m)^{1 / 2}} \\
& \times V_{1}\left(\frac{n}{q^{1 / 2}} ; t\right) V_{2}\left(\frac{a^{2} b^{4} k^{2} d^{3} m}{q^{2}} ; t, t_{f}\right) \frac{d t}{\pi},
\end{aligned}
$$

and similarly for all other combinations. We now apply the Kuznetsov formula to the completed expressions $\sum_{(*, *)} \Sigma_{1}(*, *)$ and $\sum_{(*, *)} \Sigma_{2}(*, *)$, obtaining

$$
\begin{aligned}
\Sigma(q, q)= & -\sum_{\substack{(*, *) \in\{q, 1, \mathcal{E}\}^{2} \\
(*, *) \neq(q, q)}}\left(\Sigma_{1}(*, *)-\Sigma_{2}(*, *)\right) \\
& +\sum_{q \nmid a b d k n m} \frac{\mu(d)}{(n m)^{1 / 2} a b^{2} k d^{3 / 2}} \sum_{\alpha, \beta, \gamma \in\{1,2\}} M_{\alpha}^{\beta, \gamma}
\end{aligned}
$$

where

$$
\begin{aligned}
& M_{1}^{1,1}=\delta_{n, a^{2} d m} \delta_{k, m} \mathcal{W}^{1,1}\left(\frac{n}{q^{1 / 2}}, \frac{a^{2} b^{4} k^{2} d^{3} m}{q^{2}}\right), \\
& \mathcal{W}^{1,1}(x, y)=\int_{0}^{\infty} \int_{0}^{\infty} V_{1}\left(x ; t_{2}\right) V_{2}\left(y ; t_{2}, t_{1}\right) h\left(t_{1}\right) h\left(t_{2}\right) \frac{d^{*} t_{1} d^{*} t_{2}}{2 \pi^{4}} ; \\
& M_{1}^{1,2}=\delta_{k, m} \sum_{q \mid c} \frac{1}{c} S\left(n, a^{2} d m, c\right) \mathcal{W}^{1,2}\left(\frac{n}{q^{1 / 2}}, \frac{a^{2} b^{4} k^{2} d^{3} m}{q^{2}} ; \frac{\sqrt{a^{2} d m n}}{c}\right), \\
& \mathcal{W}^{1,2}(x, y ; \eta)=\int_{0}^{\infty} \int_{0}^{\infty} V_{1}\left(x ; t_{2}\right) V_{2}\left(y ; t_{2}, t_{1}\right) h\left(t_{1}\right) h\left(t_{2}\right) \mathcal{J}^{0}\left(\eta, t_{2}\right) \frac{d^{*} t_{1} d^{*} t_{2}}{\pi^{3}} ; \\
& M_{1}^{2,1}=\delta_{n, a^{2} d m} \sum_{q \mid r} \frac{1}{r} S\left(m^{2}, k^{2}, r\right) \mathcal{W}^{2,1}\left(\frac{n}{q^{1 / 2}}, \frac{a^{2} b^{4} k^{2} d^{3} m}{q^{2}} ; \frac{k m}{r}\right), \\
& \mathcal{W}^{2,1}(x, y ; \xi)=\int_{0}^{\infty} \int_{0}^{\infty} V_{1}\left(x ; t_{2}\right) V_{2}\left(y ; t_{2}, t_{1}\right) h\left(t_{1}\right) h\left(t_{2}\right) \mathcal{J}^{+}\left(\xi, t_{1}\right) \frac{d^{*} t_{1} d^{*} t_{2}}{2 \pi^{3}} ; \\
& \quad=\sum_{q \mid c} \sum_{q \mid r} \frac{1}{c r} S\left(n, a^{2} d m, c\right) S\left(m^{2}, k^{2}, r\right) \mathcal{W}^{2,2}\left(\frac{n}{q^{1 / 2}}, \frac{a^{2} b^{4} k^{2} d^{3} m}{q^{2}} ; \frac{k m}{r}, \frac{\sqrt{a^{2} d m n}}{c}\right), \\
& \mathcal{W}^{2,2}(x, y ; \xi, \eta) \\
& =\int_{0}^{\infty} \int_{0}^{\infty} V_{1}\left(x ; t_{2}\right) V_{2}\left(y ; t_{2}, t_{1}\right) h\left(t_{1}\right) h\left(t_{2}\right) \mathcal{J}^{+}\left(\xi, t_{1}\right) \mathcal{J}^{0}\left(\eta, t_{2}\right) \frac{d^{*} t_{1} d^{*} t_{2}}{\pi^{2}} .
\end{aligned}
$$

Similarly,

$$
M_{2}^{1,1}=M_{2}^{2,1}=0
$$




$$
\begin{aligned}
& M_{2}^{1,2}= q^{1 / 2} \delta_{k, m} \sum_{q \mid c} \frac{1}{c} S\left(q n, a^{2} d m, c\right) \mathcal{W}^{1,2}\left(\frac{n}{q^{1 / 2}}, \frac{a^{2} b^{4} k^{2} d^{3} m}{q^{2}} ; \frac{\sqrt{a^{2} d m n q}}{c}\right), \\
& M_{2}^{2,2}=q^{1 / 2} \sum_{q \mid c} \sum_{q \mid r} \frac{1}{c r} S\left(q n, a^{2} d m, c\right) S\left(m^{2}, k^{2}, r\right) \\
& \times \mathcal{W}^{2,2}\left(\frac{n}{q^{1 / 2}}, \frac{a^{2} b^{4} k^{2} d^{3} m}{q^{2}} ; \frac{k m}{r}, \frac{\sqrt{a^{2} d m n q}}{c}\right) .
\end{aligned}
$$

In the rest of the paper we show that the $8+8+6=22$ (potentially) non-vanishing terms on the right hand side of (5.2) are all $O\left(q^{\varepsilon}\right)$. This will complete the proof.

\section{The contribution of the oldforms and Eisenstein series}

This section is devoted to bounding the terms $\Sigma_{1}(*, *)$ and $\Sigma_{2}(*, *)$ on the right hand side of (5.2). All terms with $(*, *) \in\{1, \mathcal{E}\}^{2}$ can easily be bounded trivially: using only the Rankin-Selberg bounds (2.6), (3.3) and the rapid decay of $V_{1}$ and $V_{2}$, we deduce

$$
\sum_{(*, *) \in\{1, \mathcal{E}\}^{2}}\left(\left|\Sigma_{1}(*, *)\right|+\left|\Sigma_{2}(*, *)\right|\right) \ll q^{-3 / 4+\varepsilon} .
$$

We proceed to bound the remaining terms $\Sigma_{1,2}(q, *)$ and $\Sigma_{1,2}(*, q)$ for $* \neq q$. The method for all these terms is identical, and we show as a typical example the case $\Sigma_{1}(q, \mathcal{E})$. By an inverse Mellin transform we have

$$
\begin{aligned}
& \Sigma_{1}(q, \mathcal{E})=\sum_{f \in \mathcal{B}_{q}} \frac{2 h\left(t_{f}\right)}{(q+1) L\left(1, \mathrm{Ad}^{2} f\right)} \int_{\mathbb{R}} \frac{h(t)}{q^{\prime \prime}\left|\zeta^{(q)}(1+2 i t)\right|^{2}} \\
& \times \int_{(2)} \int_{(2)} \prod_{ \pm}\left(L^{(q)}\left(1 / 2+u \pm i t, \operatorname{Ad}^{2} f \times g\right) \zeta^{(q)}(1 / 2+v \pm i t)\right) \\
& \times \widehat{V}_{1}(v ; t) \widehat{V}_{2}\left(u ; t, t_{f}\right) q^{2 u+v / 2} \frac{d u d v}{(2 \pi i)^{2}} \frac{d t}{\pi} .
\end{aligned}
$$

We shift both contours to $\Re u=\Re v=\varepsilon$ and use the convexity bound $L\left(s, \operatorname{Ad}^{2} f \times g\right.$ ) $\ll q^{1+\varepsilon}$ in $\Re s>1 / 2$ (note that the poles of the zeta-function at $v=1 / 2 \pm i t$ do not contribute by (3.5)). This yields the desired bound $\Sigma_{1}(q, \mathcal{E}) \ll q^{\varepsilon}$. The other three terms require only notational changes.

\section{The weight functions}

In this technical section we provide useful bounds for the weight functions $\mathcal{W}$ occurring in the definition of the quantities $M_{\alpha}^{\beta, \gamma}$. We start by collecting standard bounds for Bessel functions. The power series expansion implies

$$
e^{-\pi|t|} J_{2 i t}(x), e^{-\pi|t|} I_{2 i t}(x) \ll_{\Im t}(1+|t|)^{-1 / 2+2 \Im t} x^{-2 \Im t}, \quad x \leq 1, t \in \mathbb{C} .
$$


The asymptotic expansion implies

$$
\mathcal{J}^{+}(x, t)=\frac{1}{\sqrt{x}} e\left(\frac{x}{2 \pi}\right) v_{+}(x)+\frac{1}{\sqrt{x}} e\left(-\frac{x}{2 \pi}\right) v_{-}(x), \quad t \in \mathbb{R}, x \geq(1+|t|)^{3},
$$

where $v_{ \pm}$are smooth functions (depending on $t$ ) that satisfy $v_{ \pm}^{(j)}(x) \ll_{j} x^{-j}$ uniformly in $t$. For $j \in \mathbb{N}_{0}$ we have the general uniform upper bounds

$$
\begin{aligned}
& \frac{\partial^{j}}{\partial x^{j}} \mathcal{J}^{+}(x, t) \ll_{j}(1+|t|)^{2}\left(1+x^{-j}\right) x^{-1 / 2}, \quad x>0, t \in \mathbb{R}, \\
& \frac{\partial^{j}}{\partial x^{j}} \mathcal{J}^{-}(x, t) \ll_{j, \varepsilon}\left(1+x^{-j}\right) \times \begin{cases}x^{-\varepsilon}, & x<1+10|t|, t \in \mathbb{R}, \\
e^{-x / 2}, & x>1+10|t|, t \in \mathbb{R} .\end{cases}
\end{aligned}
$$

These bounds are not optimal, but suffice for our application.

Our first simple result shows that $\mathcal{W}$ is rapidly decreasing near $\infty$ in the first two variables and rapidly decreasing near 0 in the other variables.

Lemma 5. The following uniform bounds hold for fixed $i, j \in \mathbb{N}_{0}$ :

$$
\begin{aligned}
\mathcal{W}^{1,1}(x, y) & \ll(1+x)^{-A_{1}}(1+y)^{-A_{1}}, \\
\mathcal{W}^{1,2}(x, y ; \xi) & \ll(1+x)^{-A_{1}}(1+y)^{-A_{1}} \min \left(\xi^{-1 / 2}, \xi^{4 A_{2}}\right), \\
\mathcal{W}^{2,1}(x, y ; \eta) & \ll(1+x)^{-A_{1}}(1+y)^{-A_{1}} \min \left(\eta^{-1 / 2}, \eta^{4 A_{2}}\right), \\
\mathcal{W}^{2,2}(x, y ; \xi, \eta) & \ll(1+x)^{-A_{1}}(1+y)^{-A_{1}} \min \left(\xi^{-1 / 2}, \xi^{4 A_{2}}\right) \min \left(\eta^{-1 / 2}, \eta^{4 A_{2}}\right) .
\end{aligned}
$$

Proof. This follows directly by inserting the bounds from Lemma 1. If $\xi$ and/or $\eta$ are greater than 1, we use (7.3); if $\xi$ and/or $\eta$ are less than 1, we write the corresponding $t$-integral by symmetry as an integral over the whole real line, shift the contour down to $\Im t=-2 A_{2}$ (not crossing any poles) and use (7.1).

We will also need the following more technical result.

Lemma 6. Let $N, M, Q, X \geq 1 / 2$, and let $B \in \mathbb{N}$ and $\varepsilon>0$ be fixed (but arbitrary). Let $\rho_{1}, \rho_{2}, \alpha_{1}, \alpha_{2}>0$ and $\alpha_{3} \in \mathbb{R}$ be real numbers and let $z, z_{1}, z_{2} \in \mathbb{R}$. Let $w_{1}, w_{2}$ be two fixed smooth weight functions with support in [1,2]. Then we have uniform bounds

$$
\int_{\mathbb{R}} w_{1}\left(\frac{x}{N}\right) \mathcal{W}^{1,2}\left(\rho_{1} x, y ; \sqrt{x} \alpha_{1}\right) e(-x z) d x \ll_{B} N\left(\sqrt{N} \alpha_{1}\right)^{-1 / 2}\left(1+Q^{2} \frac{|z| \sqrt{N}}{\alpha_{1}}\right)^{-B}
$$

whenever $\alpha_{1} \sqrt{N} \geq 1 / Q$ and $y>0$, and ${ }^{2}$

\footnotetext{
${ }^{2}$ Here the term $1 /\left|\alpha_{3}\right|$ should be left out if $\alpha_{3}=0$, or one applies the convention $\min (x, \infty)=x$.
} 


$$
\begin{aligned}
\int_{\mathbb{R}} \int_{\mathbb{R}} w_{1}\left(\frac{x}{N}\right) w_{2}\left(\frac{y}{M}\right) \mathcal{W}^{2,2}\left(\rho_{1} x, \rho_{2} y ; \alpha_{1} y, \alpha_{2} \sqrt{y x}\right) e\left(-x z_{1}\right) e\left(\alpha_{3} y\right) e\left(-y z_{2}\right) d x d y \\
\ll_{B, \varepsilon} \frac{X N^{1 / 2}}{\alpha_{1}^{1 / 2} \alpha_{2}}\left(1+Q^{2} \frac{\left|z_{1}\right| \sqrt{N}}{\alpha_{2} \sqrt{M}}\right)^{-B}\left(1+Q^{2}\left|z_{2}\right| \min \left(\frac{1}{\alpha_{1}}, \frac{1}{\left|\alpha_{3}\right|}, \frac{\sqrt{M}}{\alpha_{2} \sqrt{N}}\right)\right)^{-B} \\
\quad+M N X^{-B}
\end{aligned}
$$

whenever

$$
\min \left(\alpha_{1} M, \alpha_{2} \sqrt{N M}\right) \geq 1 / Q, \quad X \geq 10+\left(\alpha_{2} \sqrt{N M}\right)^{\varepsilon},
$$

and

$$
\begin{aligned}
\int_{\mathbb{R}} w_{2}\left(\frac{y}{M}\right) \mathcal{W}^{2,2}\left(x, \rho_{2} y ; \alpha_{1} y, \alpha_{2} \sqrt{y}\right) e(-y z) d y & \\
& \ll_{B, \varepsilon} \begin{cases}\frac{M^{1 / 4}}{\left(\alpha_{1} \alpha_{2}\right)^{1 / 2}}\left(1+Q^{2}|z| \min \left(\frac{1}{\alpha_{1}}, \frac{\sqrt{M}}{\alpha_{2}}\right)\right)^{-B}, & |z| \leq \alpha_{1}, \\
\frac{X}{\alpha_{1}^{1 / 2} \alpha_{2}}\left(1+Q^{2}|z| \min \left(\frac{1}{\alpha_{1}}, \frac{\sqrt{M}}{\alpha_{2}}\right)\right)^{-B}+M X^{-B}, & |z| \geq \alpha_{1} .\end{cases}
\end{aligned}
$$

whenever

$$
\min \left(\alpha_{1} M, \alpha_{2} \sqrt{M}\right) \geq 1 / Q, \quad X \geq 10+\left(\alpha_{2} \sqrt{M}\right)^{\varepsilon}
$$

and $x>0$.

Remark. We will later apply this with $Q=X=q^{\varepsilon}$, so as a first approximation the reader can ignore the terms $Q^{2}$ and $M N X^{-B}$.

Proof. All three bounds depend on partial integration. We will always integrate the exponential factor containing $z, z_{1}, z_{2}$ respectively, and differentiate all other factors.

In order to prove (7.4), we estimate trivially using (7.3), or we integrate by parts $B$ times and then estimate trivially using (7.3). Note that each integration by parts introduces an additional factor

$$
\frac{1}{|z|}\left(\frac{1}{N}+\frac{\alpha_{1}}{\sqrt{N}}\right)\left(1+\frac{1}{\alpha_{1} \sqrt{N}}\right) \leq \frac{1}{|z|}\left((Q+1) \frac{\alpha_{1}}{\sqrt{N}}\right)(1+Q) \ll Q^{2} \frac{\alpha_{1}}{|z| \sqrt{N}} .
$$

The same strategy in the situation of (7.5) yields

$$
\frac{N^{3 / 4} M^{1 / 4}}{\left(\alpha_{1} \alpha_{2}\right)^{1 / 2}}\left(1+Q^{2} \frac{\left|z_{1}\right| \sqrt{N}}{\alpha_{2} \sqrt{M}}\right)^{-B}\left(1+Q^{2}\left|z_{2}\right| \min \left(\frac{1}{\alpha_{1}}, \frac{1}{\left|\alpha_{3}\right|}, \frac{\sqrt{M}}{\alpha_{2} \sqrt{N}}\right)\right)^{-B} .
$$

This bound suffices if $\left(\alpha_{2} \sqrt{N M}\right)^{1 / 2} \leq X$. Let us now assume that

$$
T:=\left(\alpha_{2} \sqrt{N M}\right)^{1 / 2} / X \geq 1
$$

Then $T^{\varepsilon} \leq X^{1 / 2}$ by (7.6). If

$$
Q^{2} \frac{\left|z_{1}\right| \sqrt{N}}{\alpha_{2} \sqrt{M}} \geq T^{\varepsilon / B} \quad \text { or } \quad Q^{2}\left|z_{2}\right| \min \left(\frac{1}{\alpha_{1}}, \frac{1}{\left|\alpha_{3}\right|}, \frac{\sqrt{M}}{\alpha_{2} \sqrt{N}}\right) \geq T^{\varepsilon / B},
$$


then we can replace $B$ by $B+B / \varepsilon$ in (7.8), arriving at (7.5). Let us now assume that (7.9) does not hold. Then

$$
\left(1+Q^{2} \frac{\left|z_{1}\right| \sqrt{N}}{\alpha_{2} \sqrt{M}}\right)^{-B}\left(1+Q^{2}\left|z_{2}\right| \min \left(\frac{1}{\alpha_{1}}, \frac{1}{\left|\alpha_{3}\right|}, \frac{\sqrt{M}}{\alpha_{2} \sqrt{N}}\right)\right)^{-B} \geq T^{-2 \varepsilon} \geq X^{-1},
$$

hence we only need to prove the upper bound $N^{1 / 2} /\left(\alpha_{2} \alpha_{1}^{1 / 2}\right)+M N X^{-B}$ for the double integral in (7.5). Compared to the trivial estimate in (7.8) with $B=0$ we need to save a factor $\left(\alpha_{2} \sqrt{N M}\right)^{1 / 2}$. This comes from a standard stationary phase type argument. For convenience, we give precise details. We split the $t_{2}$-integral in the definition of $\mathcal{W}^{2,2}$ into two pieces: $\left|t_{2}\right| \leq X^{2 / 3}$ and $\left|t_{2}\right| \geq X^{2 / 3}$. For large $t_{2}$, we estimate the $t_{1}, t_{2}$-integrals, as well as the above $x, y$-integral, trivially using the rapid decay of the weight function $h$. This contributes the second term on the right hand side of (7.5). For small $t_{2}$, we split $\mathcal{J}^{0}=\frac{1}{2}\left(\mathcal{J}^{+}+\mathcal{J}^{-}\right)$. By (7.3) we can bound the second term trivially due to the exponential decay of the Bessel $K$-function getting again a contribution that is easily majorized by $\ll_{B} M N X^{-B}$. For $\mathcal{J}^{+}$we insert the asymptotic formula (7.2). The $x$-integral then becomes (for $y \asymp M$ )

$$
\begin{aligned}
\int_{0}^{\infty} w_{1}\left(\frac{x}{N}\right) V_{1}\left(\rho_{1} x ; t_{2}\right) \frac{v_{ \pm}\left(\alpha_{2} \sqrt{x y}\right)}{\sqrt{\alpha_{2} \sqrt{x y}}} e\left( \pm \frac{\alpha_{2} \sqrt{x y}}{2 \pi}-x z_{1}\right) d x \\
=\frac{N^{3 / 4}}{\sqrt{\alpha_{2} \sqrt{y}}} \int_{0}^{\infty} W(x) e\left( \pm \beta_{1} \sqrt{x}-\beta_{2} x\right) d x
\end{aligned}
$$

say, where $W(x)=w_{1}(x) V_{1}\left(\rho_{1} N x ; t_{2}\right) v_{ \pm}\left(\alpha_{2} \sqrt{x N y}\right) x^{-1 / 2}$ is a function with support on [1,2] and bounded derivatives (uniformly in all parameters except $t_{2}$ ), and $\beta_{1}=$ $\alpha_{2} \sqrt{N y} /(2 \pi), \beta_{2}=N z_{1}$. If $\left|\beta_{2} / \beta_{1}\right| \notin\left[10^{-3}, 10^{3}\right]$, we integrate by parts sufficiently often, each time saving at least a factor $\alpha_{2} \sqrt{N y} \gg X^{2}$, and we obtain the trivial bound $O\left(M N X^{-B}\right)$. If $\left|\beta_{1}\right| \asymp\left|\beta_{2}\right|$, then another change of variables yields

$$
\frac{N^{3 / 4}}{\sqrt{\alpha_{2} \sqrt{y}}} \frac{\beta_{1}^{2}}{\beta_{2}^{2}} \int_{0}^{\infty} W\left(x \frac{\beta_{1}^{2}}{\beta_{2}^{2}}\right) e\left(\frac{\beta_{1}^{2}}{\left|\beta_{2}\right|}\left( \pm \sqrt{x}-\operatorname{sgn}\left(\beta_{2}\right) x\right)\right) d x \ll \frac{\sqrt{N}}{\sqrt{\alpha_{2} \beta_{1} \sqrt{y}}} \asymp \frac{N^{1 / 2}}{\alpha_{2} M^{1 / 2}}
$$

by a standard stationary phase argument (e.g. [St, p. 334]). Integrating trivially over $y$ produces another factor $\left(M / \alpha_{1}\right)^{1 / 2}$, and the proof of $(7.5)$ is complete in all cases.

The proof of (7.7) is almost identical, so we highlight only the key points. Integrating by parts and using (7.3) yields a preliminary bound

$$
\frac{M^{1 / 4}}{\left(\alpha_{1} \alpha_{2}\right)^{1 / 2}}\left(1+Q^{2}|z| \min \left(\frac{1}{\alpha_{1}}, \frac{\sqrt{M}}{\alpha_{2}}\right)\right)^{-B} .
$$

This is acceptable if $X \geq\left(\alpha_{2} \sqrt{M}\right)^{1 / 2}$ or if $|z| \leq \alpha_{1}$. In the other case, we argue as above, and hence we only need to show the upper bound $\left(\alpha_{1}^{1 / 2} \alpha_{2}\right)^{-1}+M X^{-B}$ for the integral in (7.7). We cut the $t_{1}, t_{2}$-integral in the definition of $\mathcal{W}^{2,2}$ according to whether $\left|t_{1}\right|$ and/or $\left|t_{2}\right|$ are greater than or less than $X^{2 / 3}$. By the rapid decay of the test function $h$, we can 
assume that both $t_{1}, t_{2}$ are small. In this range we can also replace $\mathcal{J}^{0}$ by $\mathcal{J}^{+}$because of the rapid decay of the Bessel- $K$-function. For the function $\mathcal{J}^{+}$we insert the asymptotic expansion (7.2), and are left with the $y$-integral

$\int_{0}^{\infty} w_{2}\left(\frac{y}{M}\right) V_{1}\left(x ; t_{2}\right) V_{2}\left(\rho_{1} y ; t_{1}, t_{2}\right) \frac{v_{ \pm}\left(\alpha_{1} y\right)}{\sqrt{\alpha_{1} y}} \frac{v_{ \pm}\left(\alpha_{2} \sqrt{y}\right)}{\sqrt{\alpha_{2} \sqrt{y}}} e\left( \pm \frac{\alpha_{1} y}{2 \pi} \pm \frac{\alpha_{2} \sqrt{y}}{2 \pi}-y z\right) d y$.

By our present assumption $|z| \geq \alpha_{1}$ there is no phase cancellation in $\left(z \pm \alpha_{1} /(2 \pi)\right) y$, and the same stationary phase argument for the $y$-integral followed by trivial estimates in the other integrals gives the bound (7.7) as before.

\section{Estimating character sums}

The scene has now been prepared to estimate the six potentially non-vanishing terms $M_{\alpha}^{\beta, \gamma}$ on the right hand side of (5.2). This is the heart of the proof of Theorem 1 and the most technical part.

The bound

$$
\sum_{q \nmid a b d k n m} \frac{1}{(n m)^{1 / 2} a b^{2} k d^{3 / 2}}\left(\left|M_{1}^{1,1}\right|+\left|M_{1}^{1,2}\right|+\left|M_{1}^{2,1}\right|\right) \ll q^{\varepsilon}
$$

follows easily by trivial estimations using (4.3) and the bounds from Lemma 5. The other three terms need a more careful reasoning.

\subsection{The term $M_{2}^{1,2}$}

Recall that we need to estimate

$$
\begin{aligned}
& \sum_{q \nmid a b d n m} \frac{\mu(d) M_{2}^{1,2}}{n^{1 / 2} a b^{2} m^{3 / 2} d^{3 / 2}} \\
&=q^{1 / 2} \sum_{q \nmid a b m d n} \sum_{q \mid c} \frac{\mu(d) S\left(q n, a^{2} d m, c\right)}{n^{1 / 2} a b^{2} m^{3 / 2} d^{3 / 2} c} \mathcal{W}^{1,2}\left(\frac{n}{q^{1 / 2}}, \frac{a^{2} b^{4} d^{3} m^{3}}{q^{2}} ; \frac{a \sqrt{d m n q}}{c}\right) .
\end{aligned}
$$

By the decay properties of $\mathcal{W}^{1,2}$ given in Lemma 5 we can assume $a \sqrt{d m n q} \leq q^{7 / 4+\varepsilon}$, hence $q^{2} \nmid c$. Replacing $c$ by $c q$ with $q \nmid c$, we obtain by (4.4) (up to a negligible error)

$$
-q^{-1 / 2} \sum_{q \nmid a b m d n c} \frac{\mu(d) S\left(n, a^{2} d m \bar{q}, c\right)}{n^{1 / 2} a b^{2} m^{3 / 2} d^{3 / 2} c} \mathcal{W}^{1,2}\left(\frac{n}{q^{1 / 2}}, \frac{a^{2} b^{4} d^{3} m^{3}}{q^{2}} ; \frac{a \sqrt{d m n}}{c q^{1 / 2}}\right) .
$$

A trivial estimate gives only $O\left(q^{1 / 4+\varepsilon}\right)$. To improve this, we can apply Poisson summation either in $a$ or in $n$, the latter being slightly easier. We can add the terms $q \mid n$ with a negligible error, and we insert a smooth weight $w_{1}(n / N) w_{2}(a / A) w_{3}(c / C)(n / N)^{1 / 2}$ (using a smooth partition of unity) that localizes $N \leq n \leq 2 N, A \leq a \leq 2 A$ and $C \leq c \leq 2 C$. Again by Lemma 5 we can assume

$$
A \leq \frac{q^{1+\varepsilon}}{b^{2}(d m)^{3 / 2}}, \quad C \leq \frac{A \sqrt{d m N}}{q^{1 / 2-\varepsilon}} .
$$


Thus we need to bound

$$
\begin{aligned}
& \frac{1}{q^{1 / 2} N^{1 / 2}} \sum_{q \nmid a b m d c} \frac{w_{2}(a / A) w_{3}(c / C)}{a b^{2} m^{3 / 2} d^{3 / 2} c} \\
& \quad \times\left|\sum_{n} S\left(n, a^{2} d m \bar{q}, c\right) w_{1}\left(\frac{n}{N}\right) \mathcal{W}^{1,2}\left(\frac{n}{q^{1 / 2}}, \frac{a^{2} b^{4} d^{3} m^{3}}{q^{2}} ; \frac{a \sqrt{d m n}}{c q^{1 / 2}}\right)\right| .
\end{aligned}
$$

By Lemma 2 with $\alpha=a^{2} d m \bar{q}$ and $\gamma=c$, the $n$-sum is

$$
\leq \sum_{h \neq 0}\left|\int_{0}^{\infty} w_{1}\left(\frac{x}{N}\right) \mathcal{W}^{1,2}\left(\frac{x}{q^{1 / 2}}, \frac{a^{2} b^{4} d^{3} m^{3}}{q^{2}} ; \frac{a \sqrt{d m x}}{c q^{1 / 2}}\right) e\left(-\frac{x h}{c}\right) d x\right| .
$$

The estimate (7.4) with

$$
\rho_{1}=\frac{1}{q^{1 / 2}}, \quad \alpha_{1}=\frac{a \sqrt{d m}}{c q^{1 / 2}}, \quad Q=q^{\varepsilon}
$$

(and $y=a^{2} b^{4} d^{3} m^{3} / q^{2}$ ) is applicable by (8.1) if $a \asymp A$ and $c \asymp C$, and bounds (8.3) by

$$
\begin{aligned}
q^{\varepsilon} \sum_{h \neq 0} N\left(\frac{a \sqrt{d m N}}{c q^{1 / 2}}\right)^{-1 / 2}\left(1+|h| \sqrt{N} \frac{q^{1 / 2}}{a \sqrt{d m}}\right)^{-10} \\
\\
\ll q^{\varepsilon} N\left(\frac{a \sqrt{d m N}}{c q^{1 / 2}}\right)^{-1 / 2}\left(\sqrt{N} \frac{q^{1 / 2}}{a \sqrt{d m}}\right)^{-1}=\frac{N^{1 / 4} c^{1 / 2} a^{1 / 2}(d m)^{1 / 4}}{q^{1 / 4-\varepsilon}} .
\end{aligned}
$$

We substitute this back into (8.2) getting the final bound

$$
\frac{1}{q^{3 / 4-\varepsilon} N^{1 / 4}} \sum_{a b m d c} \frac{w_{2}(a / A) w_{3}(c / C)}{(a c)^{1 / 2} b^{2}(m d)^{5 / 4}} \ll q^{\varepsilon}
$$

by $(8.1)$.

8.2. The term $M_{1}^{2,2}$

Here we need to bound

$$
\begin{aligned}
& \frac{1}{q^{2}} \sum_{q \nmid a b d k n m} \sum_{c, r} \frac{\mu(d) S\left(n, a^{2} d m, q c\right) S\left(m^{2}, k^{2}, q r\right)}{(n m)^{1 / 2} a b^{2} k d^{3 / 2} c r} \\
& \quad \times \mathcal{W}^{2,2}\left(\frac{n}{q^{1 / 2}}, \frac{a^{2} b^{4} k^{2} d^{3} m}{q^{2}} ; \frac{k m}{q r}, \frac{\sqrt{a^{2} d m n}}{q c}\right) .
\end{aligned}
$$

The key variables are then $n, m, c, r$, and the reader can safely think of the other variables as 1 . We re-include the terms $q \mid m$. By the decay properties of $\mathcal{W}^{2,2},(4.3),(4.4)$ and 
trivial estimates, these contribute $O\left(q^{-9 / 8+\varepsilon}\right)$. We can also include the terms $q \mid n$ at a negligible cost. It is convenient to include smooth weights

$$
w_{1}(n / N) w_{2}(m / M) w_{3}(c / C) w_{4}(r / R) \frac{(n m)^{1 / 2} c r}{(N M)^{1 / 2} C R}
$$

where all $w_{j}$ have support in [1,2], and the parameters $N, M, R, C \geq 1 / 2$ satisfy (cf. Lemma 5)

$$
N \leq q^{1 / 2+\varepsilon}, \quad M \leq \frac{q^{2+\varepsilon}}{a^{2} b^{4} k^{2} d^{3}}, \quad R \leq \frac{k M}{q^{1-\varepsilon}}, \quad C \leq \frac{a \sqrt{d M N}}{q^{1-\varepsilon}} .
$$

The idea is now to apply Poisson summation in the $n$ and $m$ variables. Since the $m$-sum is very long, the second application is certainly advantageous. The benefit of the first application is not immediately obvious, since it makes the $n$-sum (which is generically of length $q^{1 / 2}$ ) longer (the new $h_{1}$-sum is generically of length $q^{3 / 4}$ ). The point here is that the $n$-sum is a linear exponential sum, and hence the resulting complete double sum after both applications of Poisson simplifies a lot, which compensates the loss in length.

More formally, we now apply Lemma 2 with $\alpha=a^{2} d m$ and $\gamma=q c$ to the $n$-sum, and then apply Lemma 4 with $\beta=-a^{2} d \bar{h}_{1}$ and $\kappa=k^{2}$ to the $m$-sum. Unfortunately this introduces a zoo of new variables. As in Lemma 4 we decompose $r=f \tilde{r}, c=f \tilde{c}$ with $f=(r, c)$. Moreover, we decompose $f=f_{1} f_{2}^{2}, \tilde{r}=\tilde{r}_{1} \tilde{r}_{2}^{2}$ with $\mu\left(f_{1}\right)^{2}=\mu\left(\tilde{r}_{1}\right)^{2}=1$, so that

$$
\begin{aligned}
& c=\tilde{c} f_{1} f_{2}^{2}, \\
& r=\tilde{r}_{1} \tilde{r}_{2}^{2} f_{1} f_{2}^{2}=\frac{f_{1} \tilde{r}_{1}}{\left(f_{1}, \tilde{r}_{1}\right)^{2}} \times\left(f_{1}, \tilde{r}_{1}\right)^{2} f_{2}^{2} \tilde{r}_{2}^{2}, \quad \mu\left(\frac{f_{1} \tilde{r}_{1}}{\left(f_{1}, \tilde{r}_{1}\right)^{2}}\right)^{2}=1 .
\end{aligned}
$$

In this way we obtain the upper bound

$$
\begin{aligned}
\frac{1}{q^{2}} \sum_{q \nmid a b d k} \sum_{f_{1} f_{2} \tilde{c} \tilde{r}_{1} \tilde{r}_{2}} \frac{\left(f_{1}, \tilde{r}_{1}\right) f_{2} \tilde{r}_{2}}{a b^{2} k d^{3 / 2}(N M)^{1 / 2} C R} w_{3}\left(\frac{f_{1} f_{2}^{2} \tilde{c}}{C}\right) w_{4}\left(\frac{f_{1} f_{2}^{2} \tilde{r}_{1} \tilde{r}_{2}^{2}}{R}\right) \\
\times \sum_{\substack{h_{1}, h_{2} \in \mathbb{Z} \\
\left(h_{1}, q f_{1} f_{2} \tilde{c}\right)=1 \\
h_{1} h_{2} \equiv a^{2} d \tilde{r}_{1} \tilde{r}_{2}^{2}(\tilde{c})}} \mid \int_{\mathbb{R}} \int_{\mathbb{R}} \mathcal{W}^{2,2}\left(\frac{x}{q^{1 / 2}}, \frac{a^{2} b^{4} k^{2} d^{3} y}{q^{2}} ; \frac{k y}{q f_{1} f_{2}^{2} \tilde{r}_{1} \tilde{r}_{2}^{2}}, \frac{\sqrt{a^{2} d x y}}{q f_{1} f_{2}^{2} \tilde{c}}\right) \\
\times w_{1}\left(\frac{x}{N}\right) w_{2}\left(\frac{y}{M}\right) e\left(-\frac{x h_{1}}{q f_{1} f_{2}^{2} \tilde{c}}\right) e\left(-\frac{y h_{2}}{q \tilde{c} \tilde{r}_{1} \tilde{r}_{2}^{2} f_{1} f_{2}^{2}}\right) d x d y \mid .
\end{aligned}
$$

The bound (7.5) with

$$
\begin{aligned}
& \rho_{1}=\frac{1}{q^{1 / 2}}, \quad \rho_{2}=\frac{a^{2} b^{4} k^{2} d^{3}}{q^{2}}, \quad \alpha_{1}=\frac{k}{q f_{1} f_{2}^{2} \tilde{r}_{1} \tilde{r}_{2}^{2}}, \quad \alpha_{2}=\frac{a \sqrt{d}}{q f_{1} f_{2}^{2} \tilde{c}}, \quad \alpha_{3}=0, \\
& z_{1}=\frac{h_{1}}{q f_{1} f_{2}^{2} \tilde{c}}, \quad z_{2}=\frac{h_{2}}{q \tilde{c} \tilde{r}_{1} \tilde{r}_{2}^{2} f_{1} f_{2}^{2}}, \quad Q=X=q^{\varepsilon}
\end{aligned}
$$


is applicable by (8.4) if $f_{1} f_{2}^{2} \tilde{c} \asymp C$ and $f_{1} f_{2}^{2} \tilde{r}_{1} \tilde{r}_{2}^{2} \asymp R$ and implies that the double integral is at most

$\ll q^{\varepsilon} \frac{N^{1 / 2} q^{3 / 2} C R^{1 / 2}}{a(d k)^{1 / 2}}\left(1+\frac{\left|h_{1}\right| \sqrt{N}}{a \sqrt{d M}}\right)^{-10}\left(1+\left|h_{2}\right| \min \left(\frac{1}{k \tilde{c}}, \frac{\sqrt{M} C}{a \tilde{c} R \sqrt{d N}}\right)\right)^{-10}+q^{-100}$.

We can ignore the second term, and we sum the first term over $h_{1}, h_{2}$ getting the upper bound

$$
\ll q^{\varepsilon} \frac{N^{1 / 2} q^{3 / 2} C R^{1 / 2}}{a(d k)^{1 / 2}} \frac{a \sqrt{d M}}{\sqrt{N}}\left(k+\frac{a R \sqrt{d N}}{\sqrt{M} C}\right) .
$$

We substitute this back and sum over $f_{1}, f_{2}, \tilde{r}_{1}, \tilde{r}_{2}, \tilde{c}$ to obtain a total contribution of

$$
\begin{aligned}
\frac{1}{q^{2-\varepsilon}} \sum_{a b d k} \frac{1}{a b^{2} k d^{3 / 2}(N M)^{1 / 2}} & \frac{N^{1 / 2} q^{3 / 2} C R^{1 / 2}}{a(d k)^{1 / 2}} \frac{a \sqrt{d M}}{\sqrt{N}}\left(k+\frac{a R \sqrt{d N}}{\sqrt{M} C}\right) \\
= & \frac{1}{q^{1 / 2-\varepsilon}} \sum_{a b d k}\left(\frac{C R^{1 / 2}}{a b^{2} d^{3 / 2} k^{1 / 2} N^{1 / 2}}+\frac{R^{3 / 2}}{b^{2} d k^{3 / 2} M^{1 / 2}}\right) .
\end{aligned}
$$

We insert the upper bound for $C$ and $R$ from (8.4), then the upper bound for $M$, and obtain the desired bound $q^{\varepsilon}$.

\subsection{The term $M_{2}^{2,2}$}

We argue similarly to the previous subsection and consider the term

$$
\begin{aligned}
\frac{1}{q^{3 / 2}} \sum_{q \nmid a b d k n m} \sum_{c, r} \frac{\mu(d) S\left(q n, a^{2} d m, q c\right) S\left(m^{2}, k^{2}, q r\right)}{(n m)^{1 / 2} a b^{2} k d^{3 / 2} c r} \\
\times \mathcal{W}^{2,2}\left(\frac{n}{q^{1 / 2}}, \frac{a^{2} b^{4} k^{2} d^{3} m}{q^{2}} ; \frac{k m}{q r}, \frac{a \sqrt{d m n}}{\sqrt{q} c}\right) .
\end{aligned}
$$

First we observe that by the decay properties of $\mathcal{W}^{2,2}$ from Lemma 5 we can assume that $a \sqrt{d m n / q} \leq q^{3 / 4+\varepsilon}$, hence $q \nmid c$. We rewrite the previous display using (4.4) (up to a negligible error and up to sign) as

$$
\begin{aligned}
q^{-3 / 2} \sum_{q \nmid a b d k n m c} \sum_{r} \frac{\mu(d) S\left(n, a^{2} d m \bar{q}, c\right) S\left(m^{2}, k^{2}, q r\right)}{(n m)^{1 / 2} a b^{2} k d^{3 / 2} c r} & \\
& \times \mathcal{W}^{2,2}\left(\frac{n}{q^{1 / 2}}, \frac{a^{2} b^{4} k^{2} d^{3} m}{q^{2}} ; \frac{k m}{q r}, \frac{a \sqrt{d m n}}{\sqrt{q} c}\right) .
\end{aligned}
$$

Again the key players are the variables $n, m, r, c$. We re-introduce the terms $q \mid m$ which by (4.3) and trivial estimates brings about an error of $O\left(q^{-3 / 8+\varepsilon}\right)$. The terms $q \mid n$ can be included with a negligible error. Next we introduce smooth weights

$$
w_{1}(n / N) w_{2}(m / M) w_{3}(c / C) w_{4}(r / R) \frac{(n m)^{1 / 2} c r}{(N M)^{1 / 2} C R}
$$


where all $w_{j}$ have support in [1,2], and the parameters $N, M, R, C \geq 1 / 2$ satisfy (cf. Lemma 5)

$$
N \leq q^{1 / 2+\varepsilon}, \quad M \leq \frac{q^{2+\varepsilon}}{a^{2} b^{4} k^{2} b^{3}}, \quad R \leq \frac{k M}{q^{1-\varepsilon}}, \quad C \leq \frac{a \sqrt{d M N}}{q^{1 / 2-\varepsilon}} .
$$

There is one special case that we need to treat separately: if $c=1$, then the Kloosterman sum $S\left(n, a^{2} d m \bar{q}, c\right)$ degenerates. We will postpone this case for the moment and assume $C>1$, so that automatically $c \neq 1$. Now we apply Lemma 2 with $\gamma=c, \alpha=a^{2} d m \bar{q}$, getting

$$
\begin{aligned}
\frac{1}{q^{3 / 2}} \sum_{q \nmid a b d k c} \sum_{m, r} & \sum_{\left(h_{1}, c\right)=1} \frac{\mu(d) w_{2}(m / M) w_{3}(c / C) w_{4}(r / R)}{(N M)^{1 / 2} a b^{2} k d^{3 / 2} C R} e\left(-\frac{a^{2} d m \overline{q h_{1}}}{c}\right) S\left(m^{2}, k^{2}, q r\right) \\
& \times \int_{\mathbb{R}} w_{1}\left(\frac{x}{N}\right) \mathcal{W}^{2,2}\left(\frac{x}{q^{1 / 2}}, \frac{a^{2} b^{4} k^{2} d^{3} m}{q^{2}} ; \frac{k m}{q r}, \frac{a \sqrt{d m x}}{\sqrt{q} c}\right) e\left(-\frac{x h_{1}}{c}\right) d x .
\end{aligned}
$$

Since $c \neq 1$, we have $h_{1} \neq 0$. Hence we can use the reciprocity formula

$$
e\left(-\frac{a^{2} d m \overline{q h_{1}}}{c}\right)=e\left(\frac{a^{2} d m \bar{c}}{q h_{1}}\right) e\left(-\frac{a^{2} d m}{c q h_{1}}\right) .
$$

In order to display the similarities to the computation in the previous subsection, we switch the roles of $c$ and $h_{1}$, obtaining

$$
\begin{aligned}
& \frac{1}{q^{3 / 2}} \sum_{q \nmid a b d k} \sum_{\substack{m, r \\
c \in \mathbb{Z} \backslash\{0\}}} \sum_{\left(h_{1}, q c\right)=1} \frac{\mu(d) w_{2}(m / M) w_{3}\left(h_{1} / C\right) w_{4}(r / R)}{(N M)^{1 / 2} a b^{2} k d^{3 / 2} C R} e\left(\frac{a^{2} d m \overline{h_{1}}}{q c}\right) S\left(m^{2}, k^{2}, q r\right) \\
& \quad \times e\left(-\frac{a^{2} d m}{c q h_{1}}\right) \int_{\mathbb{R}} \mathcal{W}^{2,2}\left(\frac{x}{q^{1 / 2}}, \frac{a^{2} b^{4} k^{2} d^{3} m}{q^{2}} ; \frac{k m}{q r}, \frac{a \sqrt{d m x}}{\sqrt{q} h_{1}}\right) w_{1}\left(\frac{x}{N}\right) e\left(\frac{x c}{h_{1}}\right) d x .
\end{aligned}
$$

Having done this, we now apply Lemma 4 with $\kappa=k^{2}, \beta=a^{2} d \bar{h}_{1}$ and use the same parametrization (8.5) as in the previous estimation. Thus we arrive at the upper bound

$$
\begin{aligned}
& \frac{1}{q^{3 / 2}} \sum_{q \nmid a b d k} \sum_{\substack{\tilde{r}_{1} \\
\tilde{c} \in \tilde{r}_{2}, f_{1}, f_{2} \\
\tilde{c} \in\{0\}}} \frac{\left(f_{1}, \tilde{r}_{1}\right) f_{2} \tilde{r}_{2}}{(N M)^{1 / 2} a b^{2} k d^{3 / 2} C R} \sum_{\substack{h_{1} \in \mathbb{N}, h_{2} \in \mathbb{Z} \\
\left(h_{1}, q f_{1} f_{2}^{2} \tilde{c}\right)=1 \\
h_{1} h_{2} \equiv a^{2} d \tilde{r}_{1} \tilde{r}_{2}^{2}(\tilde{c})}} w_{3}\left(\frac{h_{1}}{C}\right) w_{4}\left(\frac{\tilde{r}_{1} \tilde{r}_{2}^{2} f_{1} f_{2}^{2}}{R}\right) \\
& \times \mid \int_{\mathbb{R}} \int_{\mathbb{R}} \mathcal{W}^{2,2}\left(\frac{x}{q^{1 / 2}}, \frac{a^{2} b^{4} k^{2} d^{3} y}{q^{2}} ; \frac{k y}{q f_{1} f_{2}^{2} \tilde{r}_{1} \tilde{r}_{2}^{2}}, \frac{a \sqrt{d y x}}{\sqrt{q} h_{1}}\right) w_{1}\left(\frac{x}{N}\right) w_{2}\left(\frac{y}{M}\right) \\
& \times e\left(-\frac{x f_{1} f_{2}^{2} \tilde{c}}{h_{1}}\right) e\left(-\frac{a^{2} d y}{\tilde{c} f_{1} f_{2}^{2} q h_{1}}\right) e\left(-\frac{y h_{2}}{q \tilde{c} \tilde{r}_{1} \tilde{r}_{2}^{2} f_{1} f_{2}^{2}}\right) d x d y \mid .
\end{aligned}
$$


The bound (7.5) with

$$
\begin{aligned}
& \rho_{1}=\frac{1}{q^{1 / 2}}, \quad \rho_{2}=\frac{a^{2} b^{4} k^{2} d^{3}}{q^{2}}, \\
& \alpha_{1}=\frac{k}{q f_{1} f_{2}^{2} \tilde{r}_{1} \tilde{r}_{2}^{2}}, \quad \alpha_{2}=\frac{a \sqrt{d}}{\sqrt{q} h_{1}}, \quad \alpha_{3}=-\frac{a^{2} d}{\tilde{c} f_{1} f_{2}^{2} q h_{1}}, \\
& z_{1}=\frac{f_{1} f_{2}^{2} \tilde{c}}{h_{1}}, \quad z_{2}=\frac{h_{2}}{q \tilde{c} \tilde{r}_{1} \tilde{r}_{2}^{2} f_{1} f_{2}^{2}}, \quad Q=X=q^{\varepsilon}
\end{aligned}
$$

is applicable by (8.6) and implies that the double integral is at most

$$
\begin{aligned}
q^{\varepsilon} \frac{N^{1 / 2} q C R^{1 / 2}}{a(d k)^{1 / 2}}\left(1+f_{1} f_{2}^{2}|\tilde{c}|\right. & \left.\frac{\sqrt{q N}}{a \sqrt{d M}}\right)^{-10} \\
& \times\left(1+\left|h_{2}\right| \min \left(\frac{1}{k|\tilde{c}|}, \frac{C \sqrt{M}}{a \sqrt{d N q} R|\tilde{c}|}, \frac{C f_{1} f_{2}^{2}}{a^{2} d R}\right)\right)^{-10},
\end{aligned}
$$

up to a negligible term $q^{-100}$. Now it is just a matter of book-keeping. The sum over $h_{2}$ is at most

$$
\ll k+\frac{a \sqrt{d N q} R}{C \sqrt{M}}+\frac{a^{2} d R}{C|\tilde{c}| f_{1} f_{2}^{2}} .
$$

We sum this over $\tilde{c}$ and then over $h_{1}$ and $\tilde{r}_{1} \tilde{r}_{2}^{2} f_{1} f_{2}^{2}$ to get

$$
C R\left(\frac{a \sqrt{d M}}{\sqrt{q N}}\left(k+\frac{a \sqrt{d N q} R}{C \sqrt{M}}\right)+\frac{a^{2} d R}{C}\right),
$$

so that the total contribution is given by

$$
\begin{aligned}
\frac{1}{q^{3 / 2-\varepsilon}} \sum_{a d b k} \frac{N^{1 / 2} q C R^{1 / 2}}{(N M)^{1 / 2}(a b d)^{2} k^{3 / 2}} & \left(\frac{a \sqrt{d M}}{\sqrt{q N}}\left(k+\frac{a \sqrt{d N q} R}{C \sqrt{M}}\right)+\frac{a^{2} d R}{C}\right) \\
= & \sum_{a d b k}\left(\frac{C R^{1 / 2}}{a b^{2} d^{3 / 2} k^{1 / 2} q N^{1 / 2}}+\frac{R^{3 / 2}}{b^{2} d k^{3 / 2}(q M)^{1 / 2}}\right) .
\end{aligned}
$$

The desired bound $q^{\varepsilon}$ follows now easily from (8.6).

It remains to treat the case $c=1$, that is,

$$
\begin{aligned}
\sum_{q \nmid a b d k} \sum_{m, n, r} \frac{w_{1}(n / N) w_{2}(m / M) w_{4}(r / R)}{q^{3 / 2}(M N)^{1 / 2} R} & \frac{\mu(d) S\left(m^{2}, k^{2}, q r\right)}{a b^{2} k d^{3 / 2}} \\
& \times \mathcal{W}^{2,2}\left(\frac{n}{q^{1 / 2}}, \frac{a^{2} b^{4} k^{2} d^{3} m}{q^{2}} ; \frac{k m}{q r}, \frac{a \sqrt{d m n}}{\sqrt{q}}\right) .
\end{aligned}
$$

where $M, N, R$ are subject to (8.6), and we may also assume $a \sqrt{d M N / q} \geq q^{-\varepsilon}$. The argument in this special case is not much different from the general case above, and a 
little easier. We apply Lemma 4 with $c=1, \beta=0$ and $\kappa=k^{2}$. Writing $r=r_{1} r_{2}^{2}$ with $\mu\left(r_{1}\right)^{2}=1$, we obtain the upper bound

$$
\begin{aligned}
& \sum_{\substack { q \nmid a b d k \\
\begin{subarray}{c}{n, r_{1}, r_{2} \\
h \in \mathbb{Z}{ q \nmid a b d k \\
\begin{subarray} { c } { n , r _ { 1 } , r _ { 2 } \\
h \in \mathbb { Z } } }\end{subarray}} \frac{w_{1}(n / N) w_{4}\left(r_{1} r_{2}^{2} / R\right) r_{2}}{q^{3 / 2}(M N)^{1 / 2} R a b^{2} k d^{3 / 2}} \\
& \quad \times \int_{0}^{\infty} w_{2}\left(\frac{y}{M}\right) \mathcal{W}^{2,2}\left(\frac{n}{q^{1 / 2}}, \frac{a^{2} b^{4} k^{2} d^{3} y}{q^{2}} ; \frac{k y}{q r_{1} r_{2}^{2}}, \frac{a \sqrt{d y n}}{\sqrt{q}}\right) e\left(-\frac{y h}{q r_{1} r_{2}^{2}}\right) d y
\end{aligned}
$$

We apply (7.7) with

$$
\rho_{2}=\frac{a^{2} b^{4} k^{2} d^{3}}{q^{2}}, \quad \alpha_{1}=\frac{k}{q r_{1} r_{2}^{2}}, \quad \alpha_{2}=\frac{a \sqrt{d n}}{\sqrt{q}}, \quad z=\frac{h}{q r_{1} r_{2}^{2}}, \quad Q=X=q^{\varepsilon},
$$

getting (up to a negligible error)

$$
\begin{aligned}
& q^{\varepsilon} \sum_{a b d k} \sum_{n, r_{1}, r_{2}} \frac{w_{1}(n / N) w_{4}\left(r_{1} r_{2}^{2} / R\right) r_{2}}{q^{3 / 2}(M N)^{1 / 2} R a b^{2} k d^{3 / 2}} \\
\times & \left(\sum_{|h| \leq k} \frac{M^{1 / 4} q^{3 / 4} R^{1 / 2}}{(a k)^{1 / 2}(d N)^{1 / 4}}+\sum_{|h| \geq k} \frac{q R^{1 / 2}}{a(d k)^{1 / 2} N^{1 / 2}}\left(1+|h| \min \left(\frac{1}{k}, \frac{\sqrt{M}}{a R \sqrt{d N q}}\right)\right)^{-10}\right) .
\end{aligned}
$$

The contribution from $|h| \leq k$ is

$$
\ll q^{\varepsilon} \sum_{a b d k} \frac{R^{1 / 2} N^{1 / 4}}{q^{3 / 4} M^{1 / 4} a^{3 / 2} k^{1 / 2} b^{2} d^{7 / 4}} \ll q^{\varepsilon-9 / 8} \sum_{k \leq q^{1+\varepsilon}} M^{1 / 4} \ll q^{\varepsilon-1 / 8}
$$

by (8.6). The contribution $|h| \geq k$ is

$$
\begin{aligned}
& \ll q^{\varepsilon} \sum_{a b d k} \frac{N^{1 / 2}}{q^{3 / 2} M^{1 / 2} a b^{2} k d^{3 / 2}}\left(\frac{q R^{1 / 2} k}{a(d k)^{1 / 2} N^{1 / 2}}+\frac{q^{3 / 2} R^{3 / 2}}{M^{1 / 2} k^{1 / 2}}\right) \\
& =q^{\varepsilon} \sum_{a b d k}\left(\frac{R^{1 / 2}}{q^{1 / 2} M^{1 / 2}(a b d)^{2} k^{1 / 2}}+\frac{N^{1 / 2} R^{3 / 2}}{M a b^{2}(k d)^{3 / 2}}\right) \ll q^{\varepsilon} .
\end{aligned}
$$

by (8.6). This completes the proof.

Acknowledgments. I would like to thank M. Young and R. Schulze-Pillot for very useful comments, and the referee for pointing out a small gap in an earlier version.

The author was supported by a Volkswagen Lichtenberg Fellowship and a Starting Grant of the European Research Council. 


\section{References}

[B11] Blomer, V.: On the central value of symmetric square $L$-functions. Math. Z. 260, 755-777 (2008) Zbl 1192.11028 MR 2443329

[B12] Blomer, V.: Subconvexity for twisted $L$-functions on GL(3). Amer. J. Math. 134, 13851421 (2012) Zbl pre06098925 MR 2975240

[BH] Blomer, V., Holowinsky, R.: Bounding sup-norms of cusp forms of large level. Invent. Math. 179, 645-681 (2010) Zbl 1243.11059 MR 2587342

[BFSP] Böcherer, S., Furusawa, M., Schulze-Pillot, R.: On the global Gross-Prasad conjecture for Yoshida liftings. In: Contributions to Automorphic Forms, Geometry, and Number Theory, Johns Hopkins Univ. Press, Baltimore, MD, 105-130 (2004) Z Zbl 1088.11036 MR 2058606

[CI] Conrey, B., Iwaniec, H.: The cubic moment of central values of automorphic $L$-functions. Ann. of Math. 151, 1175-1216 (2000) Zbl 0973.11056 MR 1779567

[DFI] Duke, W., Friedlander, J., Iwaniec, H.: The subconvexity problem for Artin $L$-functions. Invent. Math. 149, 489-577 (2002) Zbl 1056.11072 MR 1923476

[FW] Feigon, B., Whitehouse, D.: Exact averages of central values of triple product $L$-functions. Int. J. Number Theory 6, 1609-1624 (2010) Zbl 1236.11050 MR 2740724

[Go] Goldfeld, D.: Automorphic Forms and $L$-functions for the $\operatorname{Group} \operatorname{GL}(n, \mathbb{R})$. Cambridge Stud. Adv. Math. 99, Cambridge Univ. Press (2006) Zbl 1108.11039 MR 2254662

[HT] Harcos, G., Templier, N.: On the sup-norm of Maass cusp forms of large level, III. Math. Ann. 356, 209-216 (2013) Zbl pre06168464 MR 3038127

[HL] Hoffstein, J., Lockhart, P.: Coefficients of Maass forms and the Siegel zero. Ann. of Math. 140, 161-176 (1994) Zbl 0814.11032 MR 1289494

[ILS] Iwaniec, H., Luo, W., Sarnak, P.: Low lying zeros of families of $L$-functions. Publ. IHES 91, 55-131 (2000) Zbl 1012.11041 MR 1828743

[IK] Iwaniec, H., Kowalski, E.: Analytic Number Theory. Amer. Math. Soc. Colloq. Publ. 53, Amer. Math. Soc., Providence, RI (2004) Z Zb 1059.11001 MR 2061214

[IS] Iwaniec, H., Sarnak, P.: $L^{\infty}$ norms of eigenfunctions of arithmetic surfaces. Ann. of Math. 141, 301-320 (1995) Zbl 0833.11019 MR 1324136

[JS] Jacquet, H., Shalika, J.: Exterior square L-functions. In: Automorphic Forms, Shimura Varieties, and L-functions, Vol. II (Ann Arbor, MI, 1988), Perspect. Math. 11, Academic Press, Boston, MA, 143-226 (1990) Zbl 0695.10025 MR 1044830

[KS] Kim, H.: Functoriality for the exterior square of GL(4) and symmetric fourth of GL(2) (with Appendix 1 by D. Ramakrishnan; Appendix 2 by H. H. Kim and P. Sarnak). J. Amer. Math. Soc. 16, 139-183 (2003) Zbl 1018.11024 MR 1937203

[KSh] Kim, H., Shahidi, F.: Functorial products for $\mathrm{GL}_{2} \times \mathrm{GL}_{3}$ and symmetric cube for $\mathrm{GL}_{2}$ (with an appendix by C. J. Bushnell and G. Henniart). Ann. of Math. 155, 837-893 (2002) Zbl 1040.11036 MR 1923967

[La] Lapid, E.: On the nonnegativity of Rankin-Selberg $L$-functions at the center of symmetry. Int. Math. Res. Notices 2003, 65-75 Zbl 1046.11032 MR 1936579

[LR] Lapid, E., Rallis, S.: On the nonnegativity of $L(1 / 2, \pi)$ for $S O_{2 n+1}$. Ann. of Math. 157, 891-917 (2003) Zbl 1067.11026 MR 1983784

[Li] Li, X., Upper bounds on $L$-functions at the edge of the critical strip. Int. Math. Res. Notices 2010, 727-755 Zbl 1219.11136 MR 2595006

[LRS] Luo, W., Rudnick, Z., Sarnak, P.: On Selberg's eigenvalue conjecture. Geom. Funct. Anal. 5, 387-401 (1995) Zbl 0844.11038 MR 1334872

[St] Stein, E.: Harmonic Analysis: Real-Variable Methods, Orthogonality, and Oscillatory Integrals. Princeton Univ. Press (1993) Zbl 0821.42001 MR 1232192

[Wa] Watson, T.: Rankin triple products and quantum chaos. arXiv:0810.0425 